TI 2013-090/III

Tinbergen Institute Discussion Paper
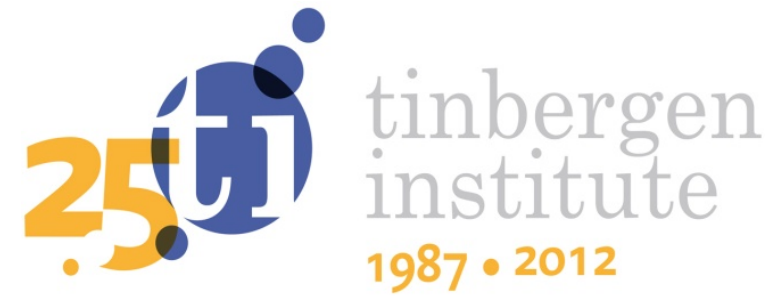

Posterior-Predictive Evidence on US

Inflation using Extended New Keynesian

Phillips Curve Models with Non-filtered

Data

Nalan Basturk1,3

Cem Cakmakli2

Pinar Ceyhan',3

Herman K. van Dijk1,3,4

' Econometric Institute, Erasmus School of Economics, Erasmus University Rotterdam;

2 Faculty of Economics and Business, University of Amsterdam;

3 Tinbergen Institute;

4 Faculty of Economics and Business Administration, VU University Amsterdam. 
Tinbergen Institute is the graduate school and research institute in economics of Erasmus University Rotterdam, the University of Amsterdam and VU University Amsterdam.

More TI discussion papers can be downloaded at http://www.tinbergen.nl

Tinbergen Institute has two locations:

Tinbergen Institute Amsterdam

Gustav Mahlerplein 117

1082 MS Amsterdam

The Netherlands

Tel.: +31(0)205251600

Tinbergen Institute Rotterdam

Burg. Oudlaan 50

3062 PA Rotterdam

The Netherlands

Tel.: +31(0)10 4088900

Fax: $+31(0) 104089031$

Duisenberg school of finance is a collaboration of the Dutch financial sector and universities, with the ambition to support innovative research and offer top quality academic education in core areas of finance.

DSF research papers can be downloaded at: http://www.dsf.nl/

Duisenberg school of finance

Gustav Mahlerplein 117

1082 MS Amsterdam

The Netherlands

Tel.: +31(0)20 5258579 


\title{
Posterior-Predictive Evidence on US Inflation using Extended New Keynesian Phillips Curve Models with non-filtered Data*
}

\author{
Nalan Basturk ${ }^{1,3}$, Cem Cakmakli ${ }^{\dagger}, 2$, Pinar Ceyhan ${ }^{1,3}$, and \\ Herman K. van Dijk $k^{1,3,4}$ \\ ${ }^{1}$ Econometric Institute, Erasmus University Rotterdam \\ ${ }^{2}$ Department of Quantitative Economics, University of Amsterdam \\ ${ }^{3}$ Tinbergen Institute \\ ${ }^{4}$ Department of Econometrics, VU University Amsterdam
}

July 2013, revised November 2013

\begin{abstract}
Changing time series properties of US inflation and economic activity, measured as marginal costs, are modeled within a set of extended New Keynesian Phillips Curve (NKPC) models. It is shown that mechanical removal or modeling of simple low frequency movements in the data may yield poor predictive results which depend on the model specification used. Basic NKPC models are extended to include structural time series models that describe typical time varying patterns in levels and volatilities. Forward and backward looking expectation components for inflation are incorporated and their relative importance is evaluated. Survey data on expected inflation are introduced to strengthen the information in the likelihood. Use is made of simulation based Bayesian techniques for the empirical analysis. No credible evidence is found on endogeneity and long run stability between inflation and marginal costs. Backward-looking inflation appears stronger than forward-looking one. Levels and volatilities of inflation are estimated more precisely using rich NKPC models. The extended NKPC structures compare favorably with existing basic Bayesian vector autoregressive and stochastic volatility models in terms of fit and prediction. Tails of the complete predictive distributions indicate an increase in the probability of deflation in recent years.
\end{abstract}

Keywords: New Keynesian Phillips curve, unobserved components, time varying parameters, level shifts, inflation expectations, survey data

JEL Classification: C11, C32, E31, E37

*A preliminary version of this paper was presented at the Tinbergen Institute workshop 'Recent Theory and Applications of DSGE Models', the Federal Reserve Bank of Atlanta workshop on 'Methods and Applications for DSGE Models', the ESOBE 2012 seminar in Vienna, the Ecares Seminar in Brussels, the CFE'12 Conference in Oviedo, the Tripartite Seminar at the Wharton School, MIT, Boston College and the Federal Reserve Bank of Chicago. We thank Gianni Amisano, Jeff Campbell, Frank DiTraglia, Sylvia Fruehwirth-Schnatter, Domenico Giannone, Jerry Hausman, James Heckman, Alejandro Justitiano, Frank Kleibergen, Michele Lenza, Richard Paap, Georgio Primicieri, Ricardo Reis and three anonymous referees and the guest-editor Frank Schorfheide for constructive discussions and very helpful comments on preliminary versions of the paper, which lead to substantial improvements. All remaining errors are the authors responsibility. Nalan Basturk and Herman K. van Dijk are supported by NWO Grant 400-09-340 and Cem Cakmakli by the AXA Research Fund.

†Nalan Basturk (basturk@ese.eur.nl), Cem Cakmakli (C.Cakmakli@uva.nl., corresponding author), Pinar Ceyhan (ceyhan@ese.eur.nl), Herman K. van Dijk (hkvandijk@ese.eur.nl) 
Tinbergen Institute is the graduate school and research institute in economics of Erasmus University Rotterdam, the University of Amsterdam and VU University Amsterdam.

More TI discussion papers can be downloaded at http://www.tinbergen.nl

Tinbergen Institute has two locations:

Tinbergen Institute Amsterdam

Gustav Mahlerplein 117

1082 MS Amsterdam

The Netherlands

Tel.: +31(0)205251600

Tinbergen Institute Rotterdam

Burg. Oudlaan 50

3062 PA Rotterdam

The Netherlands

Tel.: +31(0)10 4088900

Fax: $+31(0) 104089031$

Duisenberg school of finance is a collaboration of the Dutch financial sector and universities, with the ambition to support innovative research and offer top quality academic education in core areas of finance.

DSF research papers can be downloaded at: http://www.dsf.nl/

Duisenberg school of finance

Gustav Mahlerplein 117

1082 MS Amsterdam

The Netherlands

Tel.: +31(0)20 5258579 


\section{Introduction}

Modeling the relation between inflation and fluctuations in economic activity has been one of the building blocks of macroeconomic policy analysis. Often, the analysis of this relation, denoted as New Keynesian Phillips Curve (NKPC) models, is conducted using the short-run variations in inflation and economic activity. The conventional method for extracting this short run variation in the observed series is to demean and detrend the data prior to analysis, see Galí and Gertler (1999). However, mechanical removal of the low frequency movements in the data may lead to misspecification in the models, as suggested in Ferroni (2011) and Canova (2012) for DSGE models. The existence of complex low frequency movements, such as structural breaks and level shifts in the observed series, in particular, in the inflation series, is well documented in the literature, see McConnell and Perez-Quiros (2000) and Stock and Watson (2008). Distinct periods with different patterns can be observed for the non-filtered inflation series. The period between the early 1970s and the early 1980s is often labeled as a high inflationary period compared to earlier and later periods. A similar type of statement holds for economic activity. The real marginal cost series, often used as a proxy for economic activity, see Galí and Gertler (1999), follows a negative trend which is amplified in the recent decades. The importance of joint analysis of such high and low frequency movements in macroeconomic data has recently been documented, see Ferroni (2011), Delle Monache and Harvey (2011), Canova (2012), and Faust and Wright (2013).

In this paper we aim to contribute to this literature in four ways. We illustrate and discuss possible effects that simple prior filtering of the low frequencies in the data may have on posterior and predictive inference using a basic NKPC model. The issue is that the observed inflation and marginal cost data have more complex low frequency structures than just a simple constant mean and/or a basic linear or HP trend. We show that this misspecification affects posterior inference of the structural NKPC parameters 
and gives poor forecasting results depending on the model specification. In appendix A, we present extensive evidence on this feature using a set of simulated and real data and a range of NKPC model structures. Obviously, in well specified models and in series with relatively constant means and linear trends the misspecification effects are not severe. However, from the outset, the use of mechanical filters without properly examining the frequency features of the data is not advisable.

We extend the basic NKPC model by specifying structural time series models which allow for stochastic trends, structural breaks and stochastic volatility in inflation and log marginal costs and integrate these with the basic model. The more complex model structure enables the identification of the relation between macroeconomic variables inherent in the NKPC model, together with possible long and short run dynamics in each series.

Next, we enrich the extended NKPC models to include both forward and backward looking expectation components. There is a debate in the literature on the relative weights of these two components in explaining and forecasting inflation patterns in the U.S.. Our combined model structure can provide valuable information on that point.

As a final contribution we make use of survey data on inflation expectations from the University of Michigan Research Center, which provides quarterly one year ahead inflation expectations. It is well known that the class of NKPC models including complex time series features and basic expectation mechanisms is not easy to estimate given the usually weak data information and the few available weak instrumental variables. The proposed richer expectation mechanism and making use of survey data strengthen the likelihood information and are expected to make inference more efficient and forecasting more accurate.

Several alternatives to structural time series models for efficiently combining the NKPC model with explicit low frequency movements in the data are available. One alternative is to focus only on the high frequencies by rewriting the likelihood in the fre- 
quency domain and maximizing the (log)likelihood only over a portion of fluctuations, see e.g. Christiano and Vigfusson (2003). Another alternative is to utilize multiple prior filters, to capture possibly incorrectly specified low frequency components, see Canova and Ferroni (2011). Here we focus on explicitly modeling the low frequency movements to improve the predictive performances of the structural form models while we keep the theoretical model at a simple tractable level.

We apply the proposed set of models to quarterly U.S. data over the period 1960-I until 2012-I. For all models considered, posterior and predictive results are obtained using a simulation based Bayesian approach. Our results indicate that NKPC structures with three additional components (structural time series features, expectation mechanisms and inflation survey data) capture time variation in the low and high frequency movements of both inflation and marginal cost data. For the inflation series, the extended model identifies distinct periods with different inflation levels and volatilities. In terms of marginal costs, the local linear trend specification accommodates the smoothly changing trend observed in the series, specifically after 2000. We also find improved forecasting performance of the extended NKPC models when these are compared with basic NKPC models with demeaned and/or detrended data and with the standard stochastic volatility model proposed by Stock and Watson (2007) and, further, with an extended Bayesian Vector Autoregressive (BVAR) model which accounts for changing levels, trends and volatility in the data. The model comparison is based on predictive likelihood and Mean Squared Forecast Error (MSFE) comparisons. The Bayesian approach we adopt has additional appealing features for the models considered. In terms of inflation predictions, several measures of interest, such as deflation probabilities obtained from the lower tail of the complete predictive densities, are obtained as a by-product of Bayesian inference. Furthermore, for the most general model with good fit and forecasting features, weak endogeneity and almost non-existence of a stable long-run relationship between inflation and marginal costs can easily be assessed 
using the posterior draws of the trends and levels.

The structure of this paper is as follows: Section 2 presents the three extensions to the standard NKPC model structure. Section 3 provides the application of the proposed models and the standard NKPC model on U.S. inflation and marginal cost data. Section 4 concludes. Additional illustrations, results, details of the posterior sampling algorithms and references are provided in the appendix $\mathrm{C}$ and appendix $\mathrm{D}$.

\section{Extended New Keynesian Phillips Curve models}

We start with a standard NKPC model based on a priori filtered data. Next, we extend this model with a structural time series model in order to deal with low and high frequencies that are present in U.S. inflation and the low frequency property in the U.S. log marginal cost series. Thirdly, we extend the latter NKPC model by introducing a Hybrid NKPC model (HNKPC) with both backward and forward looking inflation expectations making use of observed inflation expectations from survey data.

The standard NKPC can be derived by the approximation of the equilibrium conditions of the firms under staggered price setting using the Calvo formulation, see Calvo (1983). The Calvo model implies that a fraction of firms optimize their prices while the remaining fraction, i.e. non-optimizing firms, keep their prices unchanged. Assuming zero inflation at the steady state the basic NKPC model derived from the firm's price setting is given as

$$
\begin{aligned}
& \tilde{\pi}_{t}=\lambda \tilde{z}_{t}+\gamma_{f} E_{t}\left(\tilde{\pi}_{t+1}\right)+\epsilon_{1, t}, \\
& \tilde{z}_{t}=\phi_{1} \tilde{z}_{t-1}+\phi_{2} \tilde{z}_{t-2}+\epsilon_{2, t}
\end{aligned}
$$

where $\tilde{\pi}_{t}$ is the filtered inflation and $\tilde{z}_{t}$ is the filtered $(\log )$ real marginal cost, $\left(\epsilon_{1, t}, \epsilon_{2, t}\right)^{\prime} \sim$ $\operatorname{NID}(0, \Sigma), \lambda$ is the slope of the Phillips curve, $\gamma_{f}$ is the weight given to the forward looking inflation, and standard stationary restrictions hold for $\left(\phi_{1}, \phi_{2}\right)$.

One way to estimate this model is to replace the expectation term with actual inflation values relying on rational expectations. Another option is to use survey data 
on expected inflation as 'observed' expectations. Still, direct substitution of survey data for expected inflation does not exploit the full model structure. In our modeling strategy, we use the full data generating process for real marginal costs together with the Phillips curve relation to form inflation expectations. ${ }^{1}$ Iterating the model forward and computing future expected inflation, model (B.1) implies that inflation can be expressed as the sum of the current and future discounted stream of the real marginal costs. Given the $\mathrm{AR}(2)$ dynamics for the long run deviation of the marginal costs, one can compute this sum and obtain a closed-form solution of model (B.1). The NKPC model takes the form of an instrumental variable model with nonlinear parameters in the inflation equation ${ }^{2}$

$$
\begin{aligned}
& \tilde{\pi}_{t}=\frac{\lambda}{1-\left(\phi_{1}+\phi_{2} \gamma_{f}\right) \gamma_{f}} \tilde{z}_{t}+\frac{\phi_{2} \gamma_{f} \lambda}{1-\left(\phi_{1}+\phi_{2} \gamma_{f}\right) \gamma_{f}} \tilde{z}_{t-1}+\epsilon_{1, t} \\
& \tilde{z}_{t}=\phi_{1} \tilde{z}_{t-1}+\phi_{2} \tilde{z}_{t-2}+\epsilon_{2, t} .
\end{aligned}
$$

One way to estimate the structural parameters is by estimating the parameters of the unrestricted reduced form model using a uniform prior and solve for the structural form parameters, see the appendix B and Kleibergen and Mavroeidis (2011) for details. However, this parameter transformation involves a complex Jacobian determinant that may seriously obscure posterior inference on the structural parameters. Hence we opt for estimating structural parameters directly.

\section{Extended NKPC models: low frequency components, non-filtered data}

We depart from the standard NKPC model by avoiding a priori data filtering and emphasize that data filtering is an integral part of modeling from an econometric point of view. Specifically, we make use of models with time varying levels as well as volatility for capturing both the low and high frequency changes in the U.S. inflation and marginal costs. Furthermore, estimating data filters together with other model parameters concerns the uncertainty related to long run specifications. Modeling the data

\footnotetext{
${ }^{1}$ We also estimate the model by inserting the survey expectations directly in model (B.1). The results are provided in the appendix $\mathrm{I}$.

${ }^{2}$ The model in (2) can be written as a triangular simultaneous equations model (SEM).
} 
filters explicitly takes this uncertainty into account while the use of filtered data does not. Finally, prior data filtering also has important effects on the predictive performance of the models as shown in section 3 .

There exists a substantial literature on the connection between actual inflation and target inflation and the firms' pricing behavior. We summarize the major issues here. In full equilibrium DSGE models with explicit monetary policy modeling, the mean level of the inflation is related to the target inflation rate. In these specifications, the target inflation rate is either assumed to be constant or is allowed to change to accommodate variation in inflation level. Prominent examples include Woodford (2003) and Sargent et al. (2006), who fix the target inflation and Erceg and Levin (2003), Schorfheide (2005), Ireland (2007) and Liu et al. (2011), who allow for discrete or continuous changes in the target inflation level.

In our partial equilibrium NKPC models, the specification of the steady state inflation level and the firms' decision process are of key importance for the final model structure. In the standard NKPC models as in Galí and Gertler (1999), assuming zero steady state inflation and keeping the prices fixed for the non-optimizing firms results in the standard form of NKPC as in model (B.1). Ascari (2004) and Ascari and Sbordone (2013), extend these models to allow for constant positive trend inflation and they analyze the implications of the trend inflation on the NKPC structure. Cogley and Sbordone (2008) take one-step further and derive the NKPC model with timevarying trend inflation modeled as a driftless random walk. Adding a trend inflation to standard NKPC assumptions, while preserving the assumption that non-optimizing firms keep their prices fixed causes the resulting NKPC coefficients to depend on the trend inflation. Thus, the interpretation of the coefficients differs from the standard model in these extended models.

Other assumptions on non-optimizing firm's pricing behaviour include indexation on past inflation (i.e. non-optimizing firms change their prices based on past inflation), 
see Smets and Wouters (2003) and Christiano et al. (2005). Alternatively, Smets and Wouters (2007) and Liu et al. (2011) make use of steady state inflation. As discussed in Ascari (2004) and Levin and Yun (2007) the structure of the NKPC remains as in model (B.1) with constant parameters if the non-optimizing firms adjust their price by the steady state inflation. Indeed, this is the route taken in Yun (1996), Jeanne (1998) and Schorfheide (2005). Moreover, Nason and Smith (2008) provide empirical evidence in favor of stable structural parameters. In our extended NKPC models with nonfiltered data we follow this assumption and keep the structural parameters constant focusing on short and long run inflation levels.

The proposed joint modeling of data filters and other model parameters is also motivated by the stylized facts for the non-filtered U.S. inflation and log marginal cost data, shown in Figure 1 over the period between 1960-I and 2012-I. ${ }^{3}$ The left panel displays distinct periods with differing inflation patterns. The period between the early 1970s and the early 1980s can be labeled as a high inflationary period with high volatility compared with the remaining periods. Existing evidence shows that the decline in inflation level and volatility is due to credible monetary policy that stabilized inflationary expectations since the early eighties, see McConnell and PerezQuiros (2000) and Stock and Watson (2007). One way to model this changing inflation behavior is to allow for regime changes in parameters, see Sims and Zha (2006) and Cogley and Sbordone (2008). We consider two cases for the inflation process. In the first case, we assume continuous level shifts in inflation using a random walk process

$$
c_{\pi, t+1}=c_{\pi, t}+\eta_{1, t+1}, \quad \eta_{1, t} \sim N I D\left(0, \sigma_{\eta_{1}}^{2}\right)
$$

-Insert Figure 1 about here-

Alternatively, we consider an inflation level subject to occasional and discrete shifts.

\footnotetext{
${ }^{3}$ Inflation is computed as the continuously compounded growth rate of the implicit GDP deflator and for the real marginal cost series we use labor share in non-farm business sector obtained from http://research.stlouisfed.org/fred2/, see Galí and Gertler (1999) for details. The right panel in Figure 1 displays real marginal costs, in natural logarithms and multiplied by 100 .
} 
Such level shifts are modeled as follows

$$
c_{\pi, t+1}=c_{\pi, t}+\kappa_{t} \eta_{1, t+1}, \quad \eta_{1, t} \sim \operatorname{NID}\left(0, \sigma_{\eta_{1}}^{2}\right),
$$

where $\kappa_{t}$ is a binary variable taking the value of 1 with probability $p_{\kappa}$ if there is level shift and the value 0 with probability $1-p_{\kappa}$ if the level does not change. This model structure allows for level shifts to depend on $p_{\kappa}$ while preserving a parsimonious model structure with only a single additional parameter. Occasional and large level shifts correspond to low values of $p_{\kappa}$ together with high values of $\sigma_{\eta_{1}}$. When $p_{\kappa}$ is 1 , the model becomes the local level model of (3). We use both specifications (3) and (4) in the empirical analysis.

The real marginal costs, shown in the right panel of Figure 1, does not exhibit discrete changes as observed in the inflation series. These data instead have a continuously changing pattern around a negative trend, which can be attributed to technology shocks. Since this trend is more prominent in the second half of the sample period, we allow for a changing trend using a local linear trend specification

$$
\begin{array}{rlrl}
c_{z, t+1} & =\mu_{z, t}+c_{z, t}+\eta_{2, t+1}, & & \eta_{2, t} \sim \operatorname{NID}\left(0, \sigma_{\eta_{2}}^{2}\right) \\
\mu_{z, t+1}=\mu_{z, t}+\eta_{3, t+1}, & \eta_{3, t} \sim N I D\left(0, \sigma_{\eta_{3}}^{2}\right) .
\end{array}
$$

This specification is flexible enough to encompass many types of filters used for detrending, see Delle Monache and Harvey (2011) and Canova (2012) for a similar specification in the more general context of DSGE models. When $\sigma_{\eta_{3}}^{2}=0$, the level of the real marginal costs follow a random walk with a drift, $\mu_{z}$. Additionally, when $\sigma_{\eta_{2}}^{2}=0$, a deterministic trend is obtained. Note that, setting only $\sigma_{\eta_{2}}^{2}=0$ but allowing $\sigma_{\eta_{3}}^{2}$ to be positive results in an integrated random walk process which can approximate nonlinear trends including the Hodrick-Prescott (HP) trend.

Together with the level specifications of the inflation and real marginal costs, the 
NKPC model in (2) using (4) and (5) takes the following form

$$
\begin{aligned}
\pi_{t}-c_{\pi, t} & =\frac{\lambda}{1-\left(\phi_{1}+\phi_{2} \gamma_{f}\right) \gamma_{f}}\left(z_{t}-c_{z, t}\right)+\frac{\phi_{2} \gamma_{f} \lambda}{1-\left(\phi_{1}+\phi_{2} \gamma_{f}\right) \gamma_{f}}\left(z_{t-1}-c_{z, t-1}\right)+\epsilon_{1, t}, \\
z_{t}-c_{z, t} & =\phi_{1}\left(z_{t-1}-c_{z, t-1}\right)+\phi_{2}\left(z_{t-2}-c_{z, t-2}\right)+\epsilon_{2, t}, \\
c_{\pi, t+1} & =c_{\pi, t}+\kappa_{t} \eta_{1, t+1}, \\
c_{z, t+1} & =\mu_{z, t}+c_{z, t}+\eta_{2, t+1}, \\
\mu_{z, t+1} & =\mu_{z, t}+\eta_{3, t+1},
\end{aligned}
$$

where $\left(\epsilon_{1, t}, \epsilon_{2, t}\right)^{\prime} \sim N I D\left(0,\left(\begin{array}{cc}\sigma_{\epsilon_{1}}^{2} & \rho \sigma_{\epsilon_{1}} \sigma_{\epsilon_{2}} \\ \rho \sigma_{\epsilon_{1}} \sigma_{\epsilon_{2}} & \sigma_{\epsilon_{2}}^{2}\end{array}\right)\right),\left(\eta_{1, t}, \eta_{2, t}, \eta_{3, t}\right)^{\prime} \sim N I D\left(0,\left(\begin{array}{ccc}\sigma_{\eta_{1}}^{2} & 0 & 0 \\ 0 & \sigma_{\eta_{2}}^{2} & 0 \\ 0 & 0 & \sigma_{\eta_{3}}^{2}\end{array}\right)\right)$ and the disturbances $\left(\epsilon_{1, t}, \epsilon_{2, t}\right)^{\prime}$ and $\left(\eta_{1, t}, \eta_{2, t}, \eta_{3, t}\right)^{\prime}$ are independent for all $t$.

\section{Adding stochastic volatility as high frequency component}

A further refinement in the NKPC model can be achieved allowing for time variation in the variances of the disturbances. This extension is particularly appealing for the inflation series, as the inflation variance changes over time substantially, see e.g. Stock and Watson (2007) for a reduced form model with a stochastic volatility component. The following state equation extends the NKPC model with a stochastic volatility process for inflation

$$
h_{t+1}=h_{t}+\eta_{4, t+1}, \eta_{4, t+1} \sim N I D\left(0, \sigma_{\eta_{4}}^{2}\right),
$$

where we specify a time-varying volatility, $\sigma_{\epsilon_{1, t}}=\exp \left(h_{t} / 2\right)$, in the first equation in (C.3). We follow Stock and Watson (2007) by fixing the value of $\sigma_{\eta_{4}}^{2}$ prior to analysis to facilitate inference. We set $\sigma_{\eta_{4}}=0.5$, which seems to work well for U.S. inflation.

An important estimation challenge in this extended model is the close relation between the changing inflation levels and volatilities. These changing data patterns can be captured by either of these model components which makes it hard to identify these components unless one makes strong prior restrictions. We fix the value of $\sigma_{\eta_{4}}^{2}$ prior to analysis to facilitate inference and in order to impose smoothness in the volatility process. It is straightforward to extend the analysis with a more flexible, strong, 
stochastic prior so that the parameter $\sigma_{\eta_{4}}^{2}$ is estimated together with the rest of the parameters. We report on this in section 4.

\section{Hybrid NKPC: forward and backward expectations using survey data} The specification of the HNKPC can be derived using an assumption on the firm's behavior, where a fraction $\omega$ of the firms, that are unable to reset their prices, adjust their price by the lagged inflation rate. The HNKPC model takes then the form of

$$
\begin{aligned}
& \tilde{\pi}_{t}=\lambda^{H} \tilde{z}_{t}+\gamma_{f}^{H} E_{t}\left(\tilde{\pi}_{t+1}\right)+\gamma_{b}^{H} \tilde{\pi}_{t-1}+\epsilon_{1, t}, \\
& \tilde{z}_{t}=\phi_{1} \tilde{z}_{t-1}+\phi_{2} \tilde{z}_{t-2}+\epsilon_{2, t},
\end{aligned}
$$

where parameters of the HNKPC model, indicated by a superscript $H$ are functions of the price stickiness parameter, a discount factor and the fraction of firms with backward looking pricing behavior. We note that the HNKPC has the same forward looking inflation expectation term in the model as the NKPC but the HNKPC has both a backward and forward looking component due to the specification of the lagged inflation deviation.

As in the NKPC case, we opt for using the full information approach by exploiting the information in the data generating process for real marginal costs. ${ }^{4}$ Iterating the first equation forward and solving for the expected inflation, the HNKPC implies the triangular simultaneous equations model which is nonlinear in parameters

$$
\begin{aligned}
\tilde{\pi}_{t} & =\frac{\lambda^{H}}{\left(1-\gamma_{b}^{H} \gamma_{f}^{H}\right)\left(1-\left(\phi_{1}+\phi_{2} \gamma_{f}^{H}\right) \gamma_{f}^{H}\right)} \tilde{z}_{t}+\frac{\phi_{2} \gamma_{f}^{H} \lambda^{H}}{\left(1-\gamma_{b}^{H} \gamma_{f}^{H}\right)\left(1-\left(\phi_{1}+\phi_{2} \gamma_{f}^{H}\right) \gamma_{f}^{H}\right)} \tilde{z}_{t-1} \\
& +\frac{\gamma_{b}^{H} \gamma_{f}^{H}}{\left(1-\gamma_{b}^{H} \gamma_{f}^{H}\right)} \sum_{k=1}^{\infty}\left(\gamma_{f}^{H}\right)^{k} E_{t}\left(\tilde{\pi}_{t+k}\right)+\frac{\gamma_{b}^{H}}{\left(1-\gamma_{b}^{H} \gamma_{f}^{H}\right)} \tilde{\pi}_{t-1}+\frac{1}{\left(1-\gamma_{b}^{H} \gamma_{f}^{H}\right)} \epsilon_{1, t} \\
\tilde{z}_{t} & =\phi_{1} \tilde{z}_{t-1}+\phi_{2} \tilde{z}_{t-2}+\epsilon_{2, t} .
\end{aligned}
$$

Unlike the NKPC solution, this system has a lagged inflation term and an infinite sum of inflation expectations. A closed form solution for the latter expression only exists under certain assumptions such as rational expectations.

We do not follow this route but proceed differently. Consider $E_{t}\left(\tilde{\pi}_{t+k}\right)=E_{t}\left(\pi_{t+k}\right)-$

\footnotetext{
${ }^{4}$ We also estimate the model by inserting the survey expectations directly in model (8). The results are provided in appendix I.
} 
$E_{t}\left(c_{\pi, t+k}\right)$ which is the difference between expected future inflation and the expected future value of the low frequency component of inflation that we modeled in (4) as a process that is similar to a random walk but subject to occasional and discrete level shifts and it has a bounded variance. One can interpret this difference as the difference between short and long run inflation expectations. As a next step we substitute the observed survey data on next period's expected inflation, denoted by $\mu_{t}$, for the expected inflation in period $t+1$, i.e. $\mu_{t}=E_{t}\left(\pi_{t+1}\right)$ and we assume the following partial adjustment mechanism

$$
\mu_{t}-c_{\pi, t+1}=\beta\left(\mu_{t-1}-c_{\pi, t}\right)+\eta_{5, t+1},
$$

where $|\beta|<1$ and $\eta_{5, t+1}$ is iid and $E_{t}\left(\eta_{5, t+1}\right)=0$. Iterating this equation forward and taking expectations one obtains $E_{t}\left(\mu_{t+k-1}-c_{\pi, t+k}\right)=\beta^{k-1}\left(\mu_{t}-c_{\pi, t+1}\right)$. That is, the partial adjustment mechanism described in (J.1) implies that the further one gets into the future the smaller will be the difference between short and long run inflation expectations. Estimates of $\beta$ will indicate the empirical speed of adjustment. For instance, for a value of the posterior mean of $\beta$ equal to 0.5 it follows that within a few periods one has almost complete adjustment. Given the restriction on $\beta$ one can solve (J.1) for $\mu_{t}$ and obtain $\mu_{t-1}=c_{\pi, t}+\sum_{j=0}^{\infty} \beta^{j} \eta_{5, t-j}$. That is, the observed survey inflation expectations are equal to the long run unobserved inflation pattern and an infinite moving average of errors with declining weights that are determined by the adjustment mechanism given in (J.1). This adaptive mechanism has a Bayesian learning and updating interpretation on the difference between short and long run expected inflation. Using this mechanism, the term $\sum_{k=1}^{\infty}\left(\gamma_{f}^{H}\right)^{k} E_{t}\left(\tilde{\pi}_{t+k}\right)$ in (9) can be 
rewritten and the HNKPC model becomes

$$
\begin{aligned}
\pi_{t}-c_{\pi, t}= & \frac{\lambda^{H}}{\left(1-\gamma_{b}^{H} \gamma_{f}^{H}\right)\left(1-\left(\phi_{1}+\phi_{2} \gamma_{f}^{H}\right) \gamma_{f}^{H}\right)}\left(z_{t}-c_{z, t}\right)+\frac{\phi_{2} \gamma_{f}^{H} \lambda^{H}}{\left(1-\gamma_{b}^{H} \gamma_{f}^{H}\right)\left(1-\left(\phi_{1}+\phi_{2} \gamma_{f}^{H}\right) \gamma_{f}^{H}\right)}\left(z_{t-1}-c_{z, t-1}\right), \\
& +\frac{\gamma_{b}^{H} \gamma_{f}^{H}}{\left(1-\gamma_{b}^{H} \gamma_{f}^{H}\right)} \frac{\gamma_{f}^{H}}{1-\gamma_{f}^{H} \beta}\left(\mu_{t}-c_{\pi, t}\right)+\frac{\gamma_{b}^{H}}{\left(1-\gamma_{b}^{H} \gamma_{f}^{H}\right)}\left(\pi_{t-1}-c_{\pi, t-1}\right)+\frac{1}{\left(1-\gamma_{b}^{H} \gamma_{f}^{H}\right)} \epsilon_{1, t}, \\
z_{t}-c_{z, t}= & \phi_{1}\left(z_{t-1}-c_{z, t-1}\right)+\phi_{2}\left(z_{t-2}-c_{z, t-2}\right)+\epsilon_{2, t} .
\end{aligned}
$$

We emphasize that alternative models on inflation expectations exist, see Mankiw et al. (2003). For instance, a skew density for $\eta_{5, t}$ allows systematic under- or overoptimism. This is an interesting topic, but outside the scope of this paper. ${ }^{5}$

Note that the model-implied expectation is for GDP inflation while the overlaid data is CPI inflation expectations. For this reason we subtract the average difference between CPI and GDP inflation from the survey data. ${ }^{6}$ Furthermore, since the survey data provide four-steps-ahead (one-year) expectations, we divide the survey data by 4 , assuming constant expectations over the year.

The NKPC model in (C.3) is a special case of (D.1) when $\gamma_{b}^{H}=0$. Then the model becomes purely forward looking. Similar to the NKPC model, we consider three case of the HNKPC model with different specifications for inflation: (i) continuous level changes; (ii) discrete occasional level changes; and (iii) discrete occasional level changes and stochastic volatility.

\section{Posterior and Predictive Evidence}

In this section we present posterior and predictive evidence on several features of the extended NKPC models using U.S. data on inflation and marginal costs. We note that the MCMC sampler for the full conditional posterior distribution is based on

\footnotetext{
${ }^{5}$ Alternatively, survey expectations may be measured with an error. In this case one can specify unobserved inflation expectations anchored around observed survey expectations. We consider this possibility and report these estimation results in appendix J. Such extensions do not seem to alter the results.

${ }^{6}$ We thank an anonymous referee for pointing this out. Our approach of recalculating the inflation expectations is similar to Del Negro and Schorfheide (2013).
} 
Gibbs sampling with a Metropolis-Hastings step and data augmentation, combining the methodologies in Geman and Geman (1984); Tanner and Wong (1987); Gerlach et al. (2000) and Çakmaklı et al. (2011). The posterior sampler, the prior distributions and a prior sensitivity analysis using prior-predictive likelihoods are given in appendix $\mathrm{H}$. We compare the results with those obtained from alternative reduced form models like BVAR models and the stochastic volatility model from Stock and Watson (2007). Specifically, we estimate two NKPC models with demeaned inflation series and with detrended real marginal costs using a linear trend or the HP filter, which are labeled NKPC-LT and NKPC-HP, respectively. In six extended NKPC models we make use of structural time series models to specify low and high frequencies. The first three of these models allow for continuous changes in the level of inflation (NKPC-TV), in addition discrete occasional level shifts (NKPC-TV-LS), and in further addition stochastic volatility for inflation (NKPC-TV-LS-SV). The final three models use the HNKPC framework with forward and backward looking expectations and using survey data. The corresponding extensions are denoted as HNKPC-TV, HNKPC-TV-LS and HNKPC-TV-LS-SV. All six models use the local linear trend specification in (C.3) for the real marginal costs. A summary of the eight models used in this paper is given in Table 1.

\section{Posterior evidence}

We display the estimation results in Table 2 and focus on four features: slope of the Phillips Curve; weight of forward and backward inflation expectations; degree of endogeneity and persistence in survey expectations. First, the slope of the NKPC $\left(\lambda^{(H)}\right)$ is estimated around 0.07 and 0.09 which is slightly higher than the conventional estimates of the Phillips curve slope, that indicate an almost flat curve, see e.g. Galí and Gertler (1999); Galí et al. (2005); Nason and Smith (2008). When we model the levels of the series explicitly, $\lambda^{(H)}$ drops to values around 0.05 for both NKPC and HNKPC models. A possible explanation for this difference is the departure from the zero steady 
state inflation assumed in the traditional NKPC models. As shown in Ascari (2004) and Ascari and Ropele (2007) among others, when firms that cannot re-optimize their prices keep their prices fixed, trend inflation can affect the slope of the NKPC. In this case, this slope is a decreasing function of the trend inflation. Still, in both NKPC and HNKPC models, the estimated slopes are substantially different from zero as point 0 is outside the $95 \%$ Highest Posterior Density Interval (HPDI) for most cases.

-Insert Table 2 about here-

Second, with respect to inflation expectations, the coefficient of the short-run inflation expectations in Table $2, \gamma_{f}^{(H)}$, is much lower than the conventional estimates, which are above 0.9 in most cases. A potential reason for this finding is the methodology used. Conventional Bayesian analyses often impose dogmatic priors on this parameter unlike our uninformative prior specification. When we consider the NKPC model with the subjective discount factor $\gamma_{f}$, the (implied) prior for the discount factor (either directly or through other parameter's priors in the steady state relations) is either fixed to the values around 0.99, see Smets and Wouters (2003) for example, or it is tightly centered around 0.99, see for example Schorfheide (2005); An and Schorfheide (2007). We also notice a relatively higher posterior standard deviation for this parameter, hence another potential cause of this finding is the relatively low information content in the data about this parameter. This is in accordance with the discussion in the appendix B on the shape of the likelihood in these macro-models. Note that the more conventional values of this parameter are still inside the 95\% HPDI.

Another reason might be the fact that, even if the models are estimated without a restriction, in most cases inflation expectations are replaced by the real leading value of the inflation relying on the rational expectations hypothesis, see e.g. Galí and Gertler (1999) and Sims (2002). However, we opt for explicitly solving for expectations resulting in a highly nonlinear system of simultaneous equations.

A striking result from Table 2 is the relative importance of the forward and backward 
looking components of the HNKPC, measured by parameters $\gamma_{f}^{H}$ and $\gamma_{b}^{H}$. On the one hand, the evidence in Galí et al. (2005) suggests a dominant forward looking effect. Cogley and Sbordone (2008) document that the forward looking component of the HNKPC model dominates once the trend variation in inflation is taken into account. Similarly, Benati (2008) shows that under stable monetary regimes with clearly defined nominal anchors, inflation appears to be (nearly) forward looking. On the other hand, many studies including Fuhrer and Moore (1995); Rudd and Whelan (2005) document a dominant backward looking effect. Our results favor the latter view since the effect of the backward looking component of inflation estimated by the HNKPC models in the bottom panel of Table 2 are substantially higher than those of the forward looking components. More specifically, Table 2 shows that the HNKPC and NKPC model results differ in terms of the forward looking components' coefficient $\gamma_{f}^{(H)}$. From an economic point of view, these results maybe driven by the model assumptions on firm behavior that differs from those of Cogley and Sbordone (2008) and Benati (2008). From an econometric point of view, as in the NKPC case, the specification of the prior distribution is crucial. In many analyses, the implied prior on these parameters suggests a support of the distribution in the interval $[0.5,1]([0,0.5])$ for $\gamma_{f}^{H}\left(\gamma_{b}^{H}\right)$, see Smets and Wouters (2003, 2007); Benati (2008); Del Negro and Schorfheide (2008) and Del Negro and Schorfheide (2013). Hence, the difference may be partly due to the presence of only one weak instrument (second order lagged marginal costs), see Nason and Smith (2008) for further empirical results and a discussion on this topic.

Third, the contemporaneous correlation between the observation disturbances determines the degree of endogeneity of the log real marginal costs in the NKPC. The estimates of this correlation parameter, $\rho$, are displayed in the fifth column of Table 2 . Posterior means of $\rho$ from all NKPC models are negative and close to 0 , with high standard deviations and point 0 is inside the $95 \%$ HPDI. For the HNKPC models, posterior means of $\rho$ are mostly positive with an even smaller magnitude. Therefore, 
the endogeneity problem does not seem to be severe and single equation inference may yield credible results for inflation and marginal costs. Still, we refrain from doing so since one neglects several cross-equation restrictions in that case.

Fourth, the $\beta$ parameter, which indicates the adaptation of the short run survey expectations to the long run inflation, has posterior means given in the fifth column of Table 2. All HNKPC models indicate relatively quick adjustment, as the posterior means are around 0.5 .

\section{Estimated Levels, Volatilities and Breaks}

We present estimated levels, trends, inflation volatilities and break probabilities for the proposed HNKPC models in Figures F.1, 3 and 4, respectively. Estimates for the NKPC counterparts are similar, and are provided in appendix C.

-Insert Figures F.1, 3, 4 about here-

The top-left panel of Figure F.1 shows estimated levels for the HNKPC-TV-LS-SV model. We first stress that models that only allow for discrete and occasional level shifts lead to smoother inflation levels compared to the model that allows for continuous level changes. Detailed results on this issue are provided in appendix D. In DSGE models, mean inflation is generally connected to the inflation target in the central bank's policy rule. Hence movements in trend inflation reflect to a large extent changes in the monetary policy target (see also Schorfheide (2005); Cogley and Sbordone (2008)). Adding stochastic volatility to the model with level shifts creates more frequent discrete changes in the inflation level, possibly reflecting the uncertainty in monetary policy target captured by volatility changes. Estimated marginal cost levels for the HNKPC-TV-LS-SV are given in the top-right panel of Figure F.1 and indicate a slightly nonlinear trend during the sample period.

Figure 3 presents estimated volatility levels for the $(\mathrm{H})$ NKPC model with level shifts and the stochastic volatility component. The stochastic volatility pattern coincides nicely with data features of the Great Moderation. The decline in inflation 
level and volatility after the 1980s is linked to credible monetary policy that stabilized inflationary expectations at a low level via commitment to a nominal anchor since the early eighties, see Ahmed et al. (2004); Stock and Watson (2007). The effect of this is also seen in the inflation levels presented in Figure F.1. This period of low volatility is replaced by a volatile period after 2005 and during the recent financial crisis. A slight difference between NKPC and HNKPC models occurs during the volatility peaks around 1975. High volatility is distributed more evenly in the HNKPC model with stochastic volatility, whereas for the NKPC counterpart, high volatility is concentrated around 1975. Peak points of estimated volatilities coincide with rapid and substantial changes in inflation.

Estimated break probabilities for the NKPC and HNKPC models with and without the stochastic volatility component are presented in Figure 4. The estimated level shift probabilities for the NKPC-TV-LS model identify four major shifts in the inflation level around 1966, 1973, 1982 and 2005. Note that the estimated shift probabilities in the NKPC-TV-LS-SV model demonstrate the complementarity of level shifts and changing volatility. The probabilities follow a similar pattern with the NKPC-TVLS model but the periods subject to level shifts are much longer. During the highly volatile periods of the 1970s, the model produces clear signals of changing inflation levels, as high volatility causes rapid changes in inflation. Accordingly, low volatility periods are characterized by mild but significant changes in inflation. This shows the complementarity of the stochastic volatility component and level shifts.

\section{Predictive Performance}

Predictive performances of the models are reported using predictive likelihoods, MSFEs and predictive densities which enable us to report the deflation probabilities.

The first metric we consider is the predictive likelihoods of all models in order to compare the density forecasts of the models. The one-step ahead predictive likelihood 
of the observation at $t_{0}+1, y_{t_{0}+1}$, conditional on the previous observations $y_{1: t_{0}}$, is

$$
f\left(y_{t_{0}+1} \mid y_{1: t_{0}}\right)=\int p\left(y_{t_{0}+1} \mid X_{t_{0}+1}, \theta\right) p\left(X_{t_{0}+1}, \theta \mid y_{1: t_{0}}\right) d X_{t_{0}+1} d \theta
$$

which can be computed by first generating $\left\{X_{t_{0}+1}\right\}_{m=1}^{M}$ for $M$ posterior draws, using the corresponding state equations. Next, the predictive likelihood of the observation at $t_{0}+1$ can be approximated by $\frac{1}{M} \sum_{m=1}^{M} p\left(y_{t_{0}+1} \mid X_{t_{0}+1}^{m}, \theta^{m}\right)$, where $p\left(y_{t_{0}+1} \mid X_{t_{0}+1}^{m}, \theta_{1: t_{0}}^{m}\right)$ is a multivariate normal density and $M$ is a sufficiently large number.

We base the MSFE and predictive likelihood comparisons on the inflation predictions. For the general case of $h \geq 1$ period ahead forecasts, the predictive density of inflation at time $t$ is calculated conditional on the inflation and marginal cost data up to time $t$, the estimated mean marginal cost values for the periods $t+1, \ldots, t+h$ and, if $h>1$, on the estimated mean inflation levels for the periods $t+1, \ldots, t+h-1$. For all models using survey expectations, predictive likelihoods are also conditioned on the observed survey expectations up to time $t$.

A feature of the predictive likelihoods is that these can be evaluated by $p\left(y_{t_{0}+1: T}\right)=$ $\prod_{t=t_{0}}^{T} f\left(y_{t+1} \mid y_{1: t}\right)$, which provides a tool to analyze the contribution of each observation at time period $t$, see Geweke and Amisano (2010). For the models with a priori demeaned and detrended data predictive likelihoods do not take into account the parameter uncertainty arising from this a priori step. We choose to calculate the predictive likelihoods this way, which is a fair replication of the literature.

Accurate point predictions of inflation are of key importance to economic agents such as investors and central banks. Therefore, we consider MSFE, computed as the mean of the sum of squares of the prediction errors. Point forecasts for inflation are defined as the mean of the predictive distribution, which is consistent with a quadratic loss function. We report MSFE for one and four period ahead forecasts in order to examine the forecasting ability of the models for longer horizons.

As a third performance criteria, we report the deflation risk indicated by each model, 
computed as the lower tail probability of the one step ahead predictive distributions.

Apart from the models considered so far, we include alternative reduced form models that are proven to have good predictive abilities. The first model is the unobserved component model proposed by Stock and Watson (2007), henceforth denoted as SW2007. This model captures the unobserved trend in inflation where both inflation and trend volatility follow a stochastic process, see SW2007 for details. The second model is an unrestricted Bayesian VAR (BVAR-SV) model with two lags and with stochastic volatility for inflation. BVAR models are one of the workhorse models used for forecasting macroeconomic series. For the sake of brevity, we do not provide details of this class of models and refer to Del Negro and Schorfheide (2013). We use the proposed 'TV' model extension in the BVAR-SV model, which allows for continuous changes in the level of inflation and a smoothly changing trend for the marginal costs. Both SW2007 and BVAR-TV-SV models are strong competitors for the models we propose. In all considered models, the data based prior distributions given in appendix $\mathrm{C}$, calculated using the full sample data, are used.

Predictive likelihoods and MSFE of the alternative models are presented in Table I.2. The likelihood contribution of each observation and the corresponding cumulative predictive likelihoods are displayed in Figure 5. We present the log predictive likelihoods of the competing models in the first column of Table I.2. These values together with Figure 5 indicate three groups of models in terms of their predictive performances. The first group of models include the conventional NKPC models with demeaned and detrended data (NKPC-LT and NKPC-HP). The second group consists of the NKPC models with time variation in inflation levels (NKPC-TV, NKPC-TVLS) together with BVAR-TV-SV and the SW2007 model. The models in the second group have much superior performance in terms of the predictive likelihood values. A second increase in the predictive likelihood values can be observed when we consider the models in the third group, namely the HNKPC models (HNKPC-TV, HNKPC- 
TV-LS, HNKPC-TV-LS-SV) and the NKPC model together with discrete level shifts and stochastic volatility for inflation (NKPC-TV-LS-SV).

-Insert Table I.2 and Figure 5 about here-

A similar clustering of models is observed when we compare model performances using the one period ahead MSFE with the exception of the BVAR-TV-SV model. BVAR-TV-SV model performs considerably better in terms of point prediction compatible with the HNKPC models.

Three main conclusions can be drawn from these findings. First, the conventional NKPC models with demeaned and detrended data (NKPC-LT and NKPC-HP) perform worse than the competing models both in terms of MSFE and in terms of the cumulative predictive likelihood metric. The difference between HNKPC and NKPC models in terms of point forecasts is less pronounced compared to the increase in precision when switching from models using demeaned and detrended data to the models that use the raw data. Hence it is important to estimate levels and trends together with the structural model parameters.

Second, the difference between the NKPC model with level shifts and stochastic volatility and the basic NKPC models is substantial. The former model delivers more accurate point predictions considering MSFE and predictive likelihood values. Thus, it is important to incorporate both high and low frequency movements in structural models. This performance increases further in the HNKPC models, which incorporate the survey data and the backward looking component.

Third, structural models perform at least as well as the strong reduced form candidates, the SW2007 and BVAR-TV-SV models. These findings are crucial since structural models deliver both structural macroeconomic information and predictive performance, whereas the reduced form models are solely designed for improving the predictive performance.

The evolution of the model performance over the forecast sample is shown in Fig- 
ure 5. An important finding from the figure is the increasing predictive performance of the HNKPC models and the models with stochastic volatility components after mid 1980s. This period is characterized by a decrease in inflation volatility during the Great Moderation, which the stochastic volatility component captures accurately. Moreover, the effect of the level shifts can be observed when we compare the NKPC-TV-LS-SV model with the SW2007 model. Much of the difference in the performance of these models can be attributed to the changes in inflation levels. This shows that the inflation process exhibits several regime changes.

The last metric we use for model comparison considers the implied deflationary risk. The left panel in Figure G.1 shows the entire density of the inflation predictions for the HNKPC-LS-SV model where the levels and trends are estimated together with the structural parameters. The mean predicted inflation is represented by the solid line, and the width of the predictive distribution is indicated by the white area under the inflation density. As expected, inflation predictions are concentrated around high (low) values during the high (low) inflationary periods. The uncertainty around the inflation predictions are also high for these periods, together with the periods when inflation is subject to a transition to low values around 1980s. When the observed inflation values are close to the zero bound, the predictive densities indicate deflationary risk.

-Insert Figure G.1 about here-

The right panel in Figure G.1 displays this deflationary risk, which is of key importance especially for policy making. The figure shows that NKPC models with a priori demeaned and detrended data do not signal any pronounced deflation risk except for the low deflation probabilities during mid 1970s and mid 1980s. However, extended NKPC and HNKPC models exploiting the high and low frequency movements produce clear signals of deflation risk and deflationary pressure during the recent recession.

Note that actual deflation only occurs around 2009 in this sample period and the models signal deflationary risk slightly later than this period. This result can be ex- 
plained by the agents' learning process. As indicated in Schorfheide (2005), if agents learn about the monetary policy changes later than the inflation level changes, the perceived target inflation in general equilibrium happens only gradually. In Schorfheide (2005), this is incorporated as Bayesian learning of the agents which is in line with the econometric assumption underlying our models. As the modeled state-space updating incorporates Bayesian learning, changes in the inflation level occur gradually and deflationary risk signals are delayed. Our models are still able to capture this deflationary pressure successfully.

We conclude this section with two remarks. First, the models we considered so far rely on the implicit assumption of the absence of a long-run cointegrating relationship. We assess whether this assumption is plausible for the U.S. data considering the HPCTV-LS-SV model, and find credible evidence that the existence of such a cointegrating relationship is very unlikely. Second, the proposed models extend the standard NKPC model in several ways. However, the superiority of the most extensive model, HNKPCTV-LS-SV, is based on all proposed model extensions jointly. Details on these results are provided in appendix $\mathrm{K}$ and appendix $\mathrm{I}$.

\section{Conclusion}

NKPC models constitute an integral part of macroeconomic models used for forecasting and policy analysis. These models are often estimated after demeaning and/or detrending the data. In this paper it is shown that mechanical removal of the low frequency movements in the data may lead to poor forecasts. Potential structural breaks and level shifts as well as changing volatility in the observed series require more complex models, which can handle these time variation together with the standard NKPC parameters. We have proposed a set of models where levels and trends of the series together with the volatility process are integrated with a structural NKPC model. Furthermore, we consider richer expectational mechanisms for the inflation series in 
enlarged Hybrid-NKPC models using survey data for inflation expectations.

The proposed models capture time variation in the low frequency movements of both inflation and marginal cost data. For the inflation series we identify three distinct periods with high and low inflation. The high inflationary period corresponds to 1970s, following a low inflationary period of 1960s. The last period starting with 1980s is characterized by low inflation levels corresponding to an annual inflation level around $2 \%$. When this model is blended with the stochastic volatility component, the level shifts can be identified even more precisely.

The use of macroeconomic information in the structural models together with the remaining high and low frequency movements in the data improves the predictive ability also compared to celebrated reduced form models, including the Bayesian VAR and the stochastic volatility model, see Stock and Watson (2007). Furthermore, modeling inflation expectations using survey data and adding stochastic volatility to the NKPC model structure improves in sample fit and out of sample predictive performance substantially. We also analyze deflation probabilities indicated by each competing model. The complete predictive densities, most notably from the enlarged models, indicate an increase in the probability of deflation in the U.S. in recent years.

Modeling forward and backward looking components of inflation has important effects on empirical results. Endogeneity and persistence do not appear to be very important empirical issues in NKPC model structures. Finally, we analyze the existence of a long-run relation between the low frequency movements of both series. No credible evidence is found on such a long run stable cointegrating relation for the U.S. series.

Given that incorporating low and high frequency movements explicitly in macroeconomic models provides additional insights for both policy analysis and more accurate predictions, we plan to enlarge the proposed model to a more general DSGE framework in future work. Another interesting possibility of future research is to combine different NKPC models using their predictive performances, which seem to be time varying. 


\section{References}

Ahmed S, Levin A, Wilson BA. 2004. Recent U.S. macroeconomic stability: Good policies, Good practices, or Good luck? The Review of Economics and Statistics 86: 824-832.

An S, Schorfheide F. 2007. Bayesian analysis of DSGE models. Econometric Reviews 26: $113-172$.

Ascari G. 2004. Staggered prices and trend inflation: Some nuisances. Review of Economic Dynamics 7: 642-667.

Ascari G, Ropele T. 2007. Optimal monetary policy under low trend inflation. Journal of Monetary Economics 54: 2568-2583.

Ascari G, Sbordone AM. 2013. The macroeconomics of trend inflation. Working papers, Federal Reserve Bank of New York Staff Reports 628, August 2013.

Basistha A, Nelson CR. 2007. New measures of the output gap based on the forwardlooking new Keynesian Phillips curve. Journal of Monetary Economics 54: 498-511.

Benati L. 2008. Investigating inflation persistence across monetary regimes. The Quarterly Journal of Economics 123: 1005-1060.

Çakmaklı C. 2012. Bayesian semiparametric dynamic nelson-siegel model. Working Paper Series 59-12, The Rimini Centre for Economic Analysis.

Çakmaklı C, Paap R, Van Dijk D. 2011. Modeling and estimation of synchronization in multistate Markov-switching models. Working Papers 11-002/4, Tinbergen Institute.

Calvo G. 1983. Staggered prices in a utility-maximizing framework. Journal of Monetary Economics 12: 383-398.

Canova F. 2012. Bridging DSGE models and the raw data. Working Papers 635, Barcelona Graduate School of Economics.

Canova F, Ferroni F. 2011. Multiple filtering devices for the estimation of cyclical DSGE models. Quantitative Economics 2: 73-98.

Carter CK, Kohn R. 1994. On Gibbs sampling for state space models. Biometrika 81: $541-553$.

Cecchetti SG, Hooper P, Kasman BC, Schoenholtz KL, Watson MW. 2007. Understanding the evolving inflation process. In US Monetary Policy Forum, volume 8.

Christiano LJ, Eichenbaum M, Evans CL. 2005. Nominal rigidities and the dynamic effects of a shock to monetary policy. Journal of Political Economy 113: 1-45.

Christiano LJ, Vigfusson RJ. 2003. Maximum likelihood in the frequency domain: the importance of time-to-plan. Journal of Monetary Economics 50: 789-815. 
Cogley T, Sbordone AM. 2008. Trend inflation, indexation, and inflation persistence in the New Keynesian Phillips curve. The American Economic Review 98: 2101-2126.

Del Negro M, Schorfheide F. 2008. Forming priors for DSGE models (and how it affects the assessment of nominal rigidities). Journal of Monetary Economics 55: $1191-1208$.

Del Negro M, Schorfheide F. 2013. DSGE model-based forecasting. In Elliott G, Timmermann A (eds.) Handbook of Economic Forecasting, volume 2 of Handbook of Economic Forecasting. Elsevier, 57-140.

Delle Monache D, Harvey AC. 2011. The effect of misspecification in models for extracting trends and cycles. Working Papers EWP 2011/013, Euroindicators.

Engle RF, Granger CWJ. 1987. Co-integration and error correction: Representation, estimation, and testing. Econometrica : 251-276.

Erceg CJ, Levin AT. 2003. Imperfect credibility and inflation persistence. Journal of Monetary Economics 50: 915-944.

Faust J, Wright JH. 2013. Forecasting inflation. In Elliott G, Timmermann A (eds.) Handbook of Economic Forecasting, volume 2 of Handbook of Economic Forecasting. Elsevier, 2-56.

Ferroni F. 2011. Trend agnostic one-step estimation of DSGE models. The B.E. Journal of Macroeconomics 11: 1-36.

Frühwirth-Schnatter S. 1994. Data augmentation and dynamic linear models. Journal of Time Series Analysis 15: 183-202.

Fuhrer J, Moore G. 1995. Inflation persistence. The Quarterly Journal of Economics 110: $127-59$.

Galí J, Gertler M. 1999. Inflation dynamics: A structural econometric analysis. Journal of Monetary Economics 44: 195-222.

Galí J, Gertler M, Lopez-Salido JD. 2005. Robustness of the estimates of the hybrid new keynesian phillips curve. Journal of Monetary Economics 52: 1107-1118.

Geman S, Geman D. 1984. Stochastic relaxations, Gibbs distributions, and the Bayesian restoration of images. IEEE Transaction on Pattern Analysis and Machine Intelligence 6: 721-741.

Gerlach R, Carter C, Kohn R. 2000. Efficient Bayesian inference for dynamic mixture models. Journal of the American Statistical Association : 819-828.

Geweke J. 2010. Complete and Incomplete Econometric Models (The Econometric and Tinbergen Institutes Lectures). Princeton University Press. 
Geweke J, Amisano G. 2010. Comparing and evaluating Bayesian predictive distributions of asset returns. International Journal of Forecasting 26: 216-230.

Giordani P, Kohn R. 2008. Efficient Bayesian inference for multiple change-point and mixture innovation models. Journal of Business \& Economic Statistics 26: 66-77.

Hastings WK. 1970. Monte Carlo sampling using Markov chains and their applications. Biometrika 57: 97-109.

Ireland PN. 2007. Changes in the Federal Reserve's inflation target: Causes and consequences. Journal of Money, Credit and Banking 39: 1851-1882.

Jeanne O. 1998. Generating real persistent effects of monetary shocks: How much nominal rigidity do we really need? European Economic Review 42: 1009-1032.

Kastner G, Frühwirth-Schnatter S. 2013. Ancillarity-sufficiency interweaving strategy (asis) for boosting MCMC estimation of stochastic volatility models. Computational Statistics \& Data Analysis .

Kim CJ, Nelson CR. 1999. State-space models with regime switching: Classical and Gibbs-Sampling approaches with applications, volume 1. The MIT Press, 1 edition.

Kim S, Shephard N, Chib S. 1998. Stochastic volatility: Likelihood inference and comparison with ARCH models. Review of Economic Studies 65: 361-393.

Kleibergen F, Mavroeidis S. 2011. Identification robust priors for Bayesian analysis in DSGE models. Manuscript.

Levin A, Yun T. 2007. Reconsidering the natural rate hypothesis in a new keynesian framework. Journal of Monetary Economics 54: 1344-1365.

Liu Z, Waggoner DF, Zha T. 2011. Sources of macroeconomic fluctuations: A regimeswitching DSGE approach. Quantitative Economics 2: 251-301.

Mankiw NG, Reis R, Wolfers J. 2003. Disagreement about inflation expectations. In NBER Macroeconomics Annual 2003, volume 18 of NBER Chapters. National Bureau of Economic Research, Inc, 209-270.

McConnell MM, Perez-Quiros G. 2000. Output fluctuations in the United States: What has changed since the early 1980's? American Economic Review 90: 1464-1476.

Metropolis N, Rosenbluth AW, Rosenbluth MN, Teller AH, Teller E. 1953. Equations of state calculations by fast computing machines. Journal of Chemical Physics 21: 1087-1092.

Nason JM, Smith GW. 2008. The New Keynesian Phillips curve: lessons from singleequation econometric estimation. Economic Quarterly 94: 361-395.

Omori Y, Chib S, Shephard N, Nakajima J. 2007. Stochastic volatility with leverage: Fast and efficient likelihood inference. Journal of Econometrics 140: 425-449. 
Ritter C, Tanner MA. 1992. Facilitating the Gibbs sampler: The Gibbs stopper and the griddy-Gibbs sampler. Journal of the American Statistical Association 87: 861-868.

Rudd J, Whelan K. 2005. New tests of the new-Keynesian Phillips curve. Journal of Monetary Economics 52: 1167-1181.

Sargent T, Williams N, Zha T. 2006. Shocks and government beliefs: The rise and fall of American inflation. American Economic Review 96: 1193-1224.

Schorfheide F. 2005. Learning and monetary policy shifts. Review of Economic Dynamics 8: 392-419.

Sims CA. 2002. Solving linear rational expectations models. Computational Economics 20: $1-20$.

Sims CA, Zha T. 2006. Were there regime switches in U.S. monetary policy? American Economic Review 96: 54-81.

Smets F, Wouters R. 2003. An estimated Dynamic Stochastic General Equilibrium model of the Euro Area. Journal of the European Economic Association 1: 11231175 .

Smets F, Wouters R. 2007. Shocks and frictions in us business cycles: A bayesian dsge approach. American Economic Review 97: 586-606.

Stock JH, Watson MW. 2007. Why has U.S. inflation become harder to forecast? Journal of Money, Credit and Banking 39: 3-33.

Stock JH, Watson MW. 2008. Phillips Curve inflation forecasts. Working Paper 14322, National Bureau of Economic Research.

Tanner MA, Wong WH. 1987. The calculation of posterior distributions by data augmentation. Journal of the American Statistical Association 82: 528-550.

Woodford M. 2003. Interest and Prices: Foundations of a Theory of Monetary Policy. Princeton University Press.

Yun T. 1996. Nominal price rigidity, money supply endogeneity, and business cycles. Journal of Monetary Economics 37: 345-370.

Zellner A, Ando T, Baştürk N, Hoogerheide L, Van Dijk HK. 2013. Bayesian analysis of instrumental variable models: Acceptance-rejection within Direct Monte Carlo. Econometric Reviews 33: 3-35. 


\section{Tables and Figures}

Table 1: Standard and extended NKPC models

\begin{tabular}{|c|c|c|}
\hline low/high & NKPC & HNKPC \\
\hline $\begin{array}{l}\text { Inf: constant level } \\
\text { RMC: linear trend }\end{array}$ & NKPC-LT & HNKPC-LT* \\
\hline $\begin{array}{l}\text { Inf: constant level } \\
\text { RMC: Hodrick-Prescott filter }\end{array}$ & NKPC-HP & HNKPC-HP* \\
\hline $\begin{array}{l}\text { Inf: time varying levels } \\
\text { RMC: local linear trend }\end{array}$ & $\begin{array}{l}\text { NKPC-TV } \\
(2)-(3)-(5) \\
\end{array}$ & $\begin{array}{c}\text { HNKPC-TV } \\
(3)-(5)-(J .1)-(D .1)\end{array}$ \\
\hline $\begin{array}{l}\text { Inf: time varying levels and switching } \\
\text { RMC: local linear trend }\end{array}$ & $\begin{array}{l}\text { NKPC-TV-LS } \\
(2)-(4)-(5) \\
\end{array}$ & $\begin{array}{l}\text { HNKPC-TV-LS } \\
(4)-(5)-(J .1)-(D .1)\end{array}$ \\
\hline $\begin{array}{l}\text { Inf: } \ldots \text { and stochastic volatility } \\
\text { RMC: local linear trend }\end{array}$ & $\begin{array}{l}\text { NKPC-TV-LS-SV } \\
(2)-(4)-(5)-(7) \\
\end{array}$ & $\begin{array}{l}\text { HNKPC-TV-LS-SV } \\
(4)-(5)-(7)-(J .1)-(D .1)\end{array}$ \\
\hline
\end{tabular}

Note: Results for the models indicated by $\left({ }^{*}\right)$ are provided in the appendix. 'Inf' ('RMC') stands for Inflation (Real Marginal Cost).

Table 2: Posterior results of alternative NKPC models

\begin{tabular}{lccccccc} 
Model & $\lambda^{(H)}$ & $\gamma_{f}^{(H)}$ & $\gamma_{b}^{H}$ & $\beta$ & $\rho$ & $\phi_{1}$ & $\phi_{2}$ \\
\hline NKPC-LT & $0.07(0.03)$ & $0.36(0.24)$ & - & - & $-0.01(0.02)$ & $0.84(0.05)$ & $0.08(0.05)$ \\
NKPC-HP & $0.10(0.05)$ & $0.43(0.27)$ & - & - & $-0.05(0.04)$ & $0.66(0.05)$ & $-0.01(0.05)$ \\
NKPC-TV & $0.06(0.03)$ & $0.39(0.25)$ & - & - & $-0.09(0.06)$ & $0.82(0.05)$ & $0.06(0.05)$ \\
NKPC-TV-LS & $0.05(0.02)$ & $0.36(0.24)$ & - & - & $-0.06(0.05)$ & $0.82(0.05)$ & $0.07(0.05)$ \\
NKPC-TV-LS-SV & $0.06(0.02)$ & $0.32(0.23)$ & - & - & $-0.02(0.07)$ & $0.87(0.05)$ & $0.10(0.05)$ \\
HNKPC-TV & $0.04(0.02)$ & $0.01(0.01)$ & $0.42(0.12)$ & $0.52(0.29)$ & $0.01(0.06)$ & $0.81(0.05)$ & $0.07(0.05)$ \\
HNKPC-TV-LS & $0.04(0.02)$ & $0.01(0.01)$ & $0.47(0.10)$ & $0.50(0.19)$ & $0.02(0.01)$ & $0.81(0.06)$ & $0.16(0.07)$ \\
HNKPC-TV-LS-SV & $0.06(0.02)$ & $0.03(0.05)$ & $0.21(0.11)$ & $0.56(0.21)$ & $-0.01(0.01)$ & $0.87(0.05)$ & $0.10(0.05)$ \\
\hline
\end{tabular}

Note: The table presents posterior means and standard deviations (in parentheses) of parameters for the competing NKPC type models estimated for quarterly inflation and real marginal costs over the period 1960-I until 2012-I. $\lambda\left(\lambda^{H}\right)$ and $\gamma_{f}\left(\gamma_{f}^{H}\right)$ are the slope of the Phillips curve and the coefficient of inflation expectations in NKPC (HNKPC) model in (2) ((D.1)). $\gamma_{b}^{H}$ is the coefficient of the backward looking component in the HNKPC model in (D.1). $H$ superscript denotes the parameters of the hybrid models while these parameters without $H$ superscript correspond to the NKPC model counterparts. $\beta$ is the autoregressive parameter for the deviation of the short run expectations from the long run, as defined in (J.1). $\rho$ is the correlation coefficient of the residuals $\epsilon_{1}$ and $\epsilon_{2} . \phi_{1}$ and $\phi_{2}$ are the autoregressive parameters for the real marginal cost specification in model (2). Posterior results are based on 40000 simulations of which the first 20000 are discarded for burn-in. Model abbreviations are as in Table 1. 
Table 3: Predictive performance of NKPC models and reduced form alternatives

\begin{tabular}{lccc} 
Model & $\begin{array}{c}\text { Cumulative } \\
(\text { Log })\end{array}$ & $\begin{array}{c}\text { Mred. Likelihood } \\
\text { 1 period ahead }\end{array}$ & $\begin{array}{c}\text { MSFE } \\
\text { 4 period ahead }\end{array}$ \\
\hline SW2007 & -78.03 & 0.17 & 0.25 \\
BVAR-TV-SV & -97.98 & 0.10 & 0.25 \\
\hline NKPC-LT & -139.33 & 0.35 & 0.36 \\
NKPC-HP & -157.19 & 0.46 & 0.37 \\
\hline NKPC-TV & -46.16 & 0.14 & 0.26 \\
NKPC-TV-LS & -61.97 & 0.14 & 0.28 \\
NKPC-TV-LS-SV & -33.48 & 0.13 & 0.21 \\
\hline HNKPC-TV & -36.38 & 0.12 & 0.28 \\
HNKPC-TV-LS & -35.05 & 0.11 & 0.24 \\
HNKPC-TV-LS-SV & -18.15 & 0.09 & 0.18 \\
\hline
\end{tabular}

Note: The table reports the predictive performances of all competing models for the prediction sample over the period 1973-II until 2012-I. 'Cumulative $(\log )$ Pred. Likelihood' stands for the sum of the natural logarithms of predictive likelihoods. 'MSFE' stands for the Mean Squared Forecast Error. Results are based on 10000 simulations of which the first 5000 are discarded for burn-in. 'SW2007' stands for the model proposed by Stock and Watson (2007), and 'BVAR-TV-SV' stands for the Bayesian VAR model with time varying levels and trends and a stochastic volatility component for the inflation equation. Remaining abbreviations are as in Table 1.

Figure 1: Inflation, inflation expectations and log real marginal cost $(\times 100)$ series over the period 1960-I until 2012-I
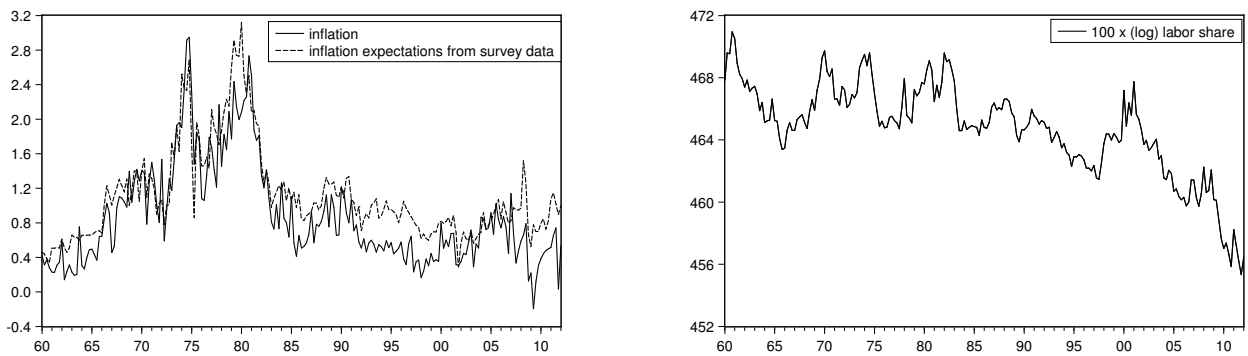
Figure 2: Level, trend and slope estimates from the HNKPC-TV-LS-SV model
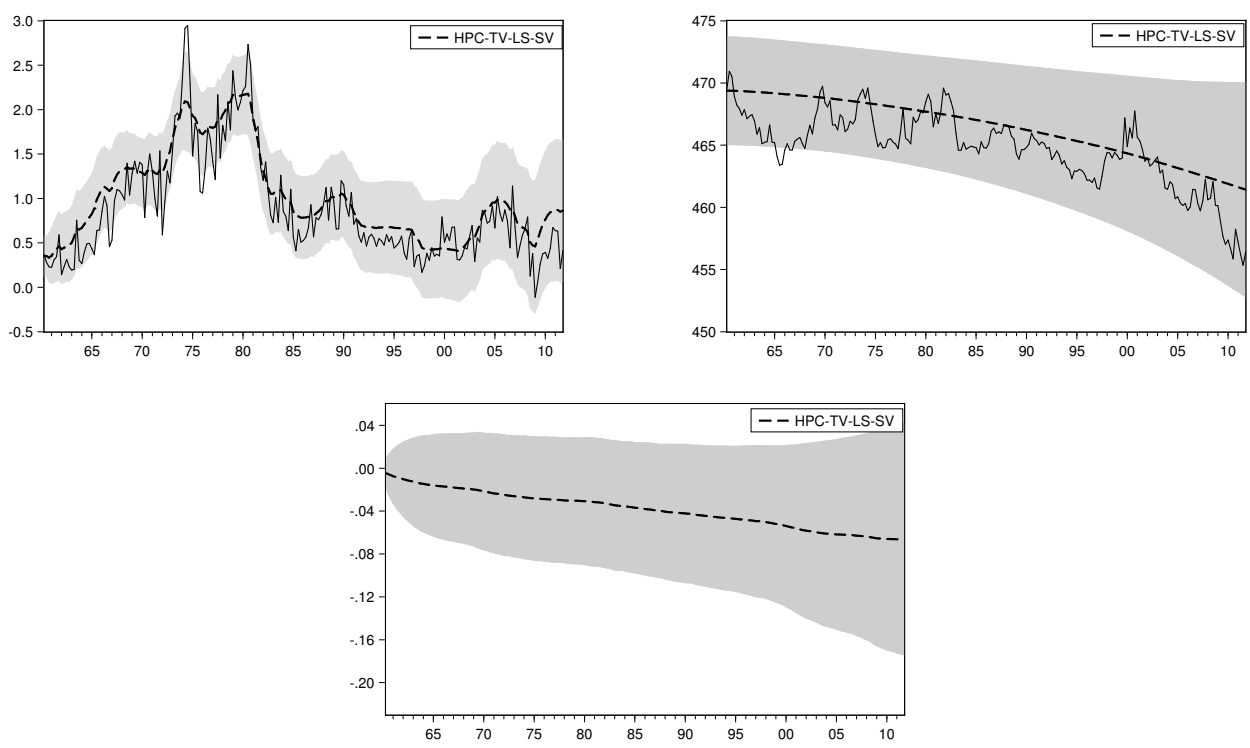

Note: The top-left panel exhibits estimated inflation levels, $c_{\pi, t}$ in model (C.3). The top-right and bottom panels show estimated (log) real marginal cost levels and the slopes, $c_{z, t}$ and $\mu_{z, t}$ in model (C.3), respectively. Grey shaded areas correspond to the 95\% HPDI. Model abbreviations are as in Table 1. However, for notational convenience we use the abbreviation ' $(\mathrm{H}) \mathrm{PC}$ ' instead of the abbreviation ' $(\mathrm{H}) \mathrm{NKPC}$ ' in the figures. Results are based on 40000 simulations of which the first 20000 are discarded for burn-in.

Figure 3: Estimated inflation volatility from the (H)NKPC-TV-LS-SV models
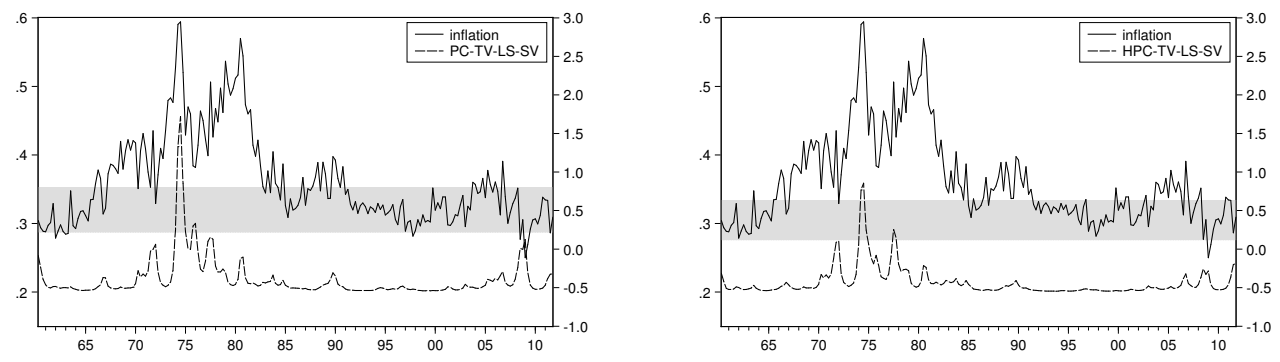

Note: The dashed and solid lines show the posterior mean of the time varying inflation volatility and the observed inflation level. The shaded areas are the 90\% HPDI of inflation volatility estimated by the equivalent models without the stochastic volatility components. Results are based on 40000 simulations of which the first 20000 are discarded for burn-in. 
Figure 4: Estimated level shift probabilities for the NKPC and HNKPC models
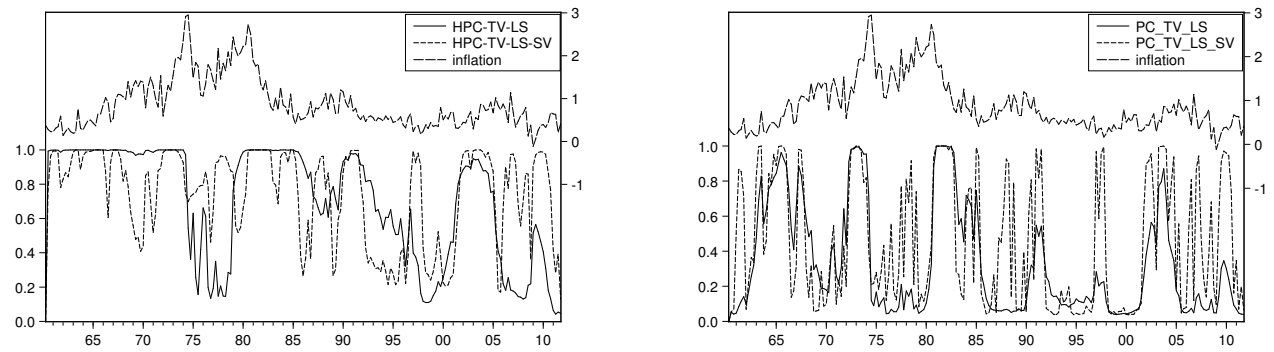

Note: The solid and dotted lines are the posterior means of the estimated level shift probabilities from the (H)NKPC-TV-LS model and the (H)NKPC-TV-LS models, respectively. The dashed line is the observed inflation level. Results are based on 40000 simulations with the first 20000 discarded for burn-in.

Figure 5: Predictive likelihoods from competing models
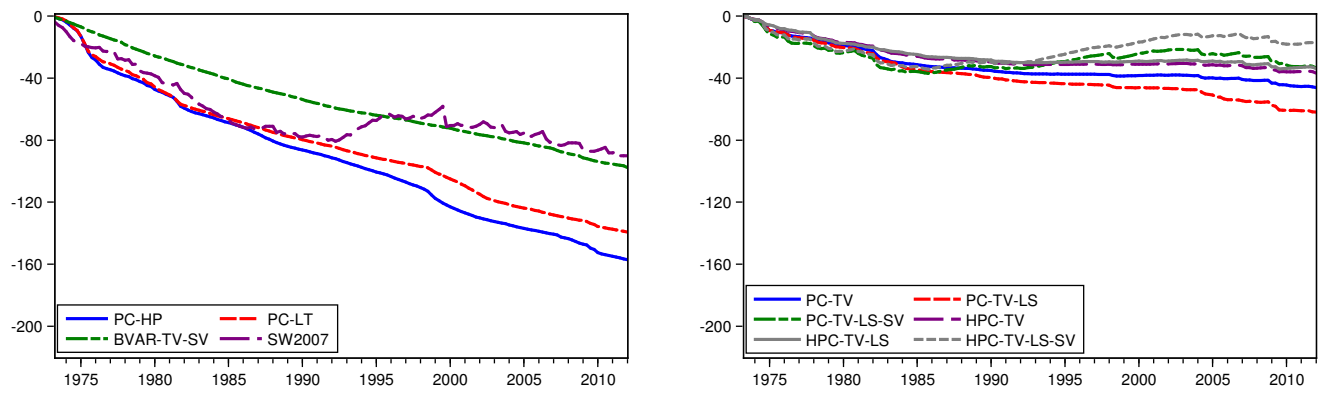

Note: The figure displays the evolution of the $(\log )$ predictive likelihoods for the computing models over the period 1973-II until 2012-I. Model abbreviations are as in Table 1. Results are based on 5000 simulations of which the first 10000 are discarded for burn-in.

Figure 6: Predicted inflation densities from HNKPC-LS-SV model and deflation probabilities implied by different Phillips curve models
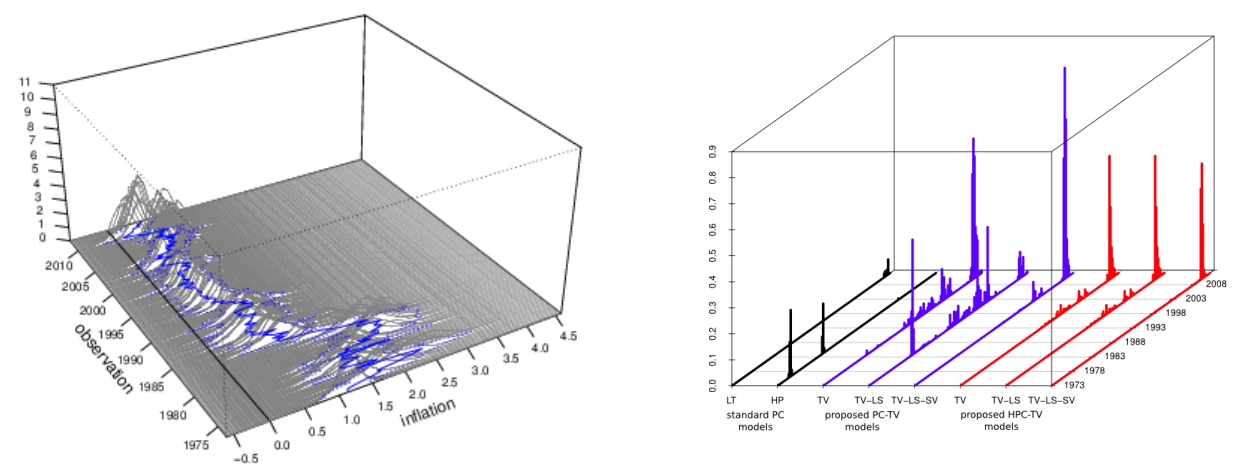

Note: The left figure presents one period ahead predictive distribution of inflation from the HNKPCLS-SV model, over the period 1973-II until 2012-I. The right figure presents deflation probabilities computed using these predictive distributions of inflation over the same period. Model abbreviations are as in Table 1. Results are based on 5000 simulations of which the first 10000 are discarded for burn-in. 


\section{Appendix}

In section A we present the effects of misspecified levels on posterior results in a standard New Keynesian Phillips Curve (NKPC). This analysis provides a straightforward motivation for the extended NKPC and HNKPC models in the paper. Specifically, we show that a priori demeaning and detrending of the data, without considering the short and long-run data properties obscure inference in these standard models.

In section $\mathrm{B}$ we elaborate and compare the inference of the NKPC model using structural form and unrestricted reduced form. This section illustrates the difficulty of inferring the unrestricted reduced form parameters and to obtain the main parameters of interest, the structural parameters, using these. This difficulty is based on the nonlinear parameter transformations required to link the structural and the reduced form models. Through simulation examples, we show that flat prior distributions used in one of the model representations can be very informative in the other model representation. This section motivates the structural parameter estimation approach we follow throughout the paper.

Sections C and D provide the details of the posterior sampling algorithms for the extended NKPC and HNKPC models proposed in the main paper. In these sections, the state space representations of the extended models and the appropriate sampling scheme are explained in detail. We further report the exact prior parameters used for the results in the paper and present a sketch of a prior sensitivity analysis based on prior-predictive likelihood comparisons.

Sections E, F and G provide posterior and predictive results for the extended NKPC and HNKPC models which are not included in the paper due to space constraints. In section E we present additional posterior and predictive results for the extended NKPC models. Main conclusions from these models are similar to the extended HNKPC model results discussed in the paper. Nonetheless, we provide these results for clarity and the 
ease of comparison. In section $\mathrm{F}$ we present additional results for the HNKPC models which are in line with the main conclusions of the paper. Section G presents the entire distribution of the inflation predictions for extended NKPC and HNKPC models we propose.

Section $\mathrm{H}$ presents the results of the prior-predictive likelihood analysis for the proposed models. The main conclusion of this section is that the adopted priors in the paper do not dominate the results. The data information is the main factor favoring the extended models we propose.

Section I presents the posterior and predictive results of the alternative NKPC and HNKPC models, considered for robustness checks, in detail. Several alternative models are compared with the extended models in the paper. We show that our main conclusions on the improved model performance through modeling the trends and levels in the data, and the use of survey data hold. We further disentangle the predictive gains from these two sources of extensions.

In section J presents a further alternative HNKPC model to the proposed HNKPC models in our paper. This model aims at accounting for the possibility of measurement errors in survey expectations. The results obtained from this alternative model are very similar to the corresponding results of in the paper, thus, we conclude that the effect of the measurement errors in survey expectations is negligible.

Section K presents a straightforward cointegration analysis for inflation and marginal cost series, based on the time-varying NKPC model structure. This analysis is performed to justify an implicit assumption in the proposed models namely the assumption that there is no stable long-run relationship between the inflation and marginal cost series. The results of this cointegration analysis are in line with the implicit assumption we make in the proposed NKPC model structures. 


\section{A Effect of misspecified level shifts on posterior es- timates of inflation persistence}

The linear NKPC captures the relation between real marginal cost $\tilde{z}_{t}$ and inflation $\tilde{\pi}_{t}$. We illustrate in this section that model misspecification resulting from ignoring level shifts in inflation data leads to overestimation of persistence in the inflation equation within a linear NKPC.

The linear NKPC model can be written as

$$
\begin{aligned}
& \tilde{\pi}_{t}=\lambda \tilde{z}_{t}+\gamma_{b} \tilde{\pi}_{t-1}+\epsilon_{1, t}, \\
& \tilde{z}_{t}=\phi_{1} \tilde{z}_{t-1}+\phi_{2} \tilde{z}_{t-2}+\epsilon_{2, t},
\end{aligned}
$$

with $\left(\epsilon_{1, t}, \epsilon_{2, t}\right)^{\prime} \sim N I D(0, \Sigma)$. This model is a triangular simultaneous equations model and can also be interpreted as an instrumental variable model with two instruments. We specify an $\mathrm{AR}(2)$ model for the marginal costs in order to mimic for the cyclical behavior of the observed series, see Basistha and Nelson (2007); Kleibergen and Mavroeidis (2011) for a similar specification. The $\mathrm{AR}(2)$ parameters are restricted to the stationary region $\left|\phi_{1}\right|+\phi_{2}<1, \quad\left|\phi_{2}\right|<1$, and the lagged adjustment parameter in the inflation equation is restricted as $0 \leq \gamma_{b}<1$. The structural parameter $\lambda$, the slope of the Phillips curve, is restricted as $0 \leq \lambda<1$ which is in line with previous evidence on the slope of the NKPC.

Since NKPC in (A.1) specifies the relation between the short-run stationary fluctuations in real marginal costs and inflation, $\tilde{\pi}_{t}$ and $\tilde{z}_{t}$ can be interpreted as the transitory components of inflation and marginal costs, in deviation from their long-run components. In fact, the observed non-filtered data can be decomposed into permanent and 
transitory components in a straightforward way as

$$
\begin{aligned}
& \pi_{t}=\tilde{\pi}_{t}+c_{\pi, t}, \\
& z_{t}=\tilde{z}_{t}+c_{z, t},
\end{aligned}
$$

where $\pi_{t}$ and $z_{t}$ are the inflation and marginal cost data, respectively, and $c_{\pi, t}$ and $c_{z, t}$ are the permanent components of the series.

In our simulation experiment, we model the steady state inflation as a constant level subject to regime shifts in order to mimic the high inflationary period during the 1970s. For modelling the permanent component of the real marginal cost series, we use a trend specification mimicking the declining real marginal cost levels in the U.S. over the sample starting from the 1960s. This specification can be formulated as follows

$$
\begin{aligned}
& c_{\pi, t}=c_{\pi, t-1}+\kappa_{t} \eta_{t-1}, \quad c_{z, t}=c_{z, t-1}+\mu_{z, t-1}, \\
& \mu_{z, t}=\mu_{z, t-1}, \quad \eta_{t} \sim N I D\left(0, \omega^{2}\right),
\end{aligned}
$$

where $\kappa_{t}$ is a binary variable indicating a level shift in the level series, $c_{\pi, t}$ and $c_{z, t}$ indicate the level value of inflation and real marginal costs, respectively, in period $t$ and $\mu_{z, t}$ is the slope of the trend in the real marginal cost series. By excluding the stochastic component for the slope and the trend of the real marginal costs in (A.3), we specify a deterministic trend for this series.

We simulate three sets of data from the model in (A.1)-(A.3). For the first set, the inflation series show no level shifts, i.e. $\kappa_{t}=0, \forall t$. For the other two sets of data, we impose different level shifts with moderate $\left(\omega^{2}=2.5\right)$ and large $\left(\omega^{2}=5\right)$ changes in the level values, respectively. For each specification we simulate 100 datasets with $T=200$ observations, where two level shifts occur in periods $t=50$ and $t=150$. The observation error variance is set to $\left(\begin{array}{cc}1 & 0.01 \\ 0.01 & 0.01\end{array}\right)$, which leads to a correlation of 0.1 between the disturbances, and parameter $\lambda$ is set to 0.1 . Note that parameters $\phi_{1}=0.1$ and $\phi_{2}=0.5$ are chosen such that the transitory component of the series is stationary. 
In order to capture the effect of model misspecification on posterior inference, when computing the transitory component, we ignore level shifts in the simulated inflation series and simply demean the series. For the marginal cost series, we remove the linear trend prior to the analysis and only focus on the effect of misspecification in the inflation series. This implies that for the simulated data with no level shifts, the model is correctly specified and the posterior results should be close to the true values. For each simulated data set we estimate the model in (A.1) using flat priors on restricted parameter regions:

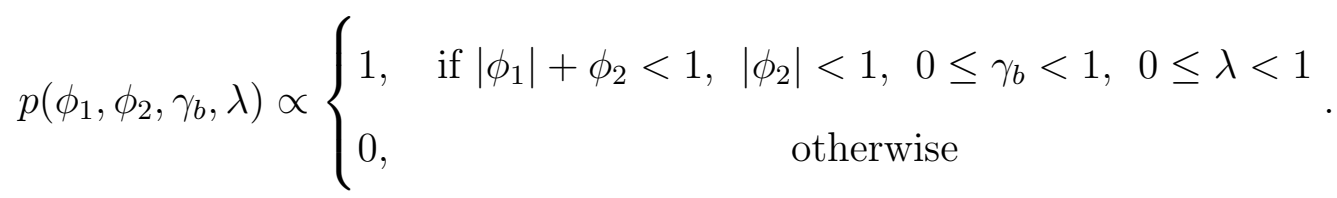

Given that model (A.1) is equivalent to an instrumental variables model with 2 instruments, it can be shown that the likelihood function for such a model combined with the flat prior on a large space yields a posterior distribution that exists but it has no first or higher moments. Due to the bounded region condition on the parameters, where the structural parameter $\lambda$ is restricted to the unit interval, all moments exist. For details, we refer to Zellner, Ando, Baştürk, Hoogerheide and Van Dijk (2013). We mention this existence result since it provides an econometric explanation why it is often difficult to estimate a structural model for macro-economic data such as (A.1). Indeed, the rather flat posterior surface plagues the inference, in particular, when $\phi_{2}$ is close to zero. Posterior moments are in our case computed by means of standard Metropolis-Hastings method on $\phi_{1}$ and $\phi_{2}$ and $\lambda$ and $\gamma_{b}$. Other Monte Carlo methods like Gibbs sampling are also feasible in this case.

Figure A.1 presents the overestimation results from 100 different simulations for each setting we consider. We report the average overestimation in posterior $\gamma_{b}$ estimates and $95 \%$ highest posterior density intervals (HPDI) for this overestimation.

The persistence parameter $\gamma_{b}$ is overestimated in all cases except for the correctly 
Figure A.1: Overestimation illustration for the backward looking NKPC model

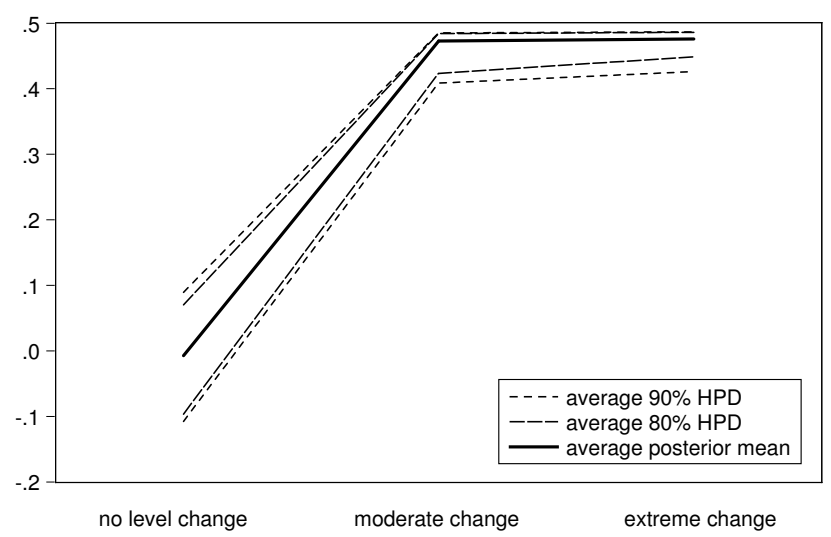

Note: The figure presents overestimation probability of parameter $\gamma_{b}$ for simulated data from the NKPC model with different structural breaks structures. We report average quantiles of overestimation based on 100 simulation replications for each parameter setting.

specified model. The degree of overestimation becomes larger with a larger shift in the level of inflation. Note that the average 95\% HPDI of overestimation becomes tighter for data with extreme changes in levels. Hence the effect of model misspecification on the persistence estimates is more pronounced if the regime shifts are extreme.

In summary, our simulation experiments using NKPC show that when the shifts in the inflation level are not modelled, inference on model persistence parameters may be severely biased due to the model misspecification. This will also hold for predictive estimates.

We note that we focused on misspecification effects on persistence measures when level shifts in the series are ignored. Similar experiments can be set up for the NKPC with weak identification (or weak instruments) by setting $\phi_{2} \approx 0$. The effect of misspecification on posterior and predictive estimates in the case of weak identification is a topic outside the scope of the present paper. We refer to Kleibergen and Mavroeidis (2011) for details on Bayesian estimation in case of weak identification. 


\section{B Structural and reduced form inference of the NKPC model}

This section presents the unrestricted reduced form inference (URF) of the NKPC model, and the inference of the corresponding structural form (SF) model parameters. The structural form (SF) representation for the basic NKPC model derived from the firm's price setting for filtered data is given as

$$
\begin{aligned}
& \tilde{\pi}_{t}=\lambda \tilde{z}_{t}+\gamma_{f} E_{t}\left(\tilde{\pi}_{t+1}\right)+\epsilon_{1, t} \\
& \tilde{z}_{t}=\phi_{1} \tilde{z}_{t-1}+\phi_{2} \tilde{z}_{t-2}+\epsilon_{2, t}
\end{aligned}
$$

where $\left(\epsilon_{1, t}, \epsilon_{2, t}\right)^{\prime} \sim N I D(0, \Sigma)$ and standard stationary restrictions hold for $\phi_{1}, \phi_{2}$.

We show that the posterior draws from the structural form parameters can be obtained using the reduced form representation of (B.1):

$$
\begin{aligned}
& \tilde{\pi}_{t}=\alpha_{1} \tilde{z}_{t-1}+\alpha_{2} \tilde{z}_{t-2}+\epsilon_{1, t}, \\
& \tilde{z}_{t}=\phi_{1} \tilde{z}_{t-1}+\phi_{2} \tilde{z}_{t-2}+\epsilon_{2, t},
\end{aligned}
$$

where $\left(\epsilon_{1, t}, \epsilon_{2, t}\right)^{\prime} \sim N I D(0, \Sigma)$, and the restricted reduced form $(\mathrm{RRF})$ representation is obtained by introducing the following restrictions on parameters in (B.1):

$$
\alpha_{1}=\frac{\lambda\left(\phi_{1}+\gamma \phi_{2}\right)}{1-\gamma\left(\phi_{1}+\gamma \phi_{2}\right)}, \quad \alpha_{2}=\frac{\lambda \phi_{2}}{1-\gamma\left(\phi_{1}+\gamma \phi_{2}\right)} .
$$

Finally, the model in (B.1) is related to an Instrumental Variables (IV) model with exact identification. Bayesian estimation of the unrestricted reduced form model in (B.2) is straightforward under flat or conjugate priors. Given the posterior draws of reduced form parameters, posterior draws of structural form parameters in (B.1) can be obtained using the transformation in (B.3). This nonlinear transformation, however, causes difficulties in setting the priors in an adequate way. The determinant of the 
Jacobian of this nonlinear transformation is $|J|=\frac{\lambda \phi_{2}^{2}}{\left(1-\gamma\left(\phi_{1}+\gamma \phi_{2}\right)\right)^{2}}$, where the Jacobian is non-zero and finite if $\gamma\left(\phi_{1}+\gamma \phi_{2}\right) \neq 1, \phi_{2} \neq 0$ and $\lambda \neq 0 .{ }^{7}$

Figure B.1 illustrates the nonlinear transformation for the SF and RRF representations, for a grid of parameter values from SF representations, and plot the corresponding RRF parameter values, and vice versa. The top panel in Figure B.1 shows the transformations from SF to RRF. Reduced form parameters $\alpha_{1}$ and $\alpha_{2}$ tend to infinity when persistence in inflation and marginal cost series are high, i.e. when the structural form parameters $\lambda$ and $\phi_{1}+\phi_{2}$ tend to 1 . The bottom panel in Figure B.1 shows the RRF to SF transformations. The corresponding SF parameters lead to an irregular shape, for example, when the instrument $z_{t-2}$ has no explanatory power with $\phi_{2}=0$ or when $\alpha_{2}=0$.

\section{Bayesian inference of the extended NKPC model}

In this section we summarize the prior specifications, our use of prior predictive likelihoods, and the posterior sampling algorithms for the extended NKPC and HNKPC models. We further present a prior sensitivity analysis for the proposed models using a prior-predictive analysis.

\section{C.1 Prior specification for parameters}

The extended NKPC and HNKPC models contain several additional parameters compared to the standard NKPC model. We classify the model parameters in five groups, and assign independent priors for each group. The first group includes the common parameters in the NKPC and HNKPC models, $\theta_{N}=\left\{\lambda, \gamma_{f}, \phi_{1}, \phi_{2}, \Sigma\right\}$, in (B.1). For the structural parameters $\left\{\lambda, \gamma_{f}, \phi_{1}, \phi_{2}\right\}$ we define flat priors on restricted regions, which also ensure that the autoregressive parameters, $\phi_{1}$ and $\phi_{2}$, are in the stationary region

\footnotetext{
${ }^{7}$ We only consider the transformation from $\left\{\lambda, \gamma, \phi_{1}, \phi_{2}\right\}$ to $\left\{\alpha_{1}, \alpha_{2}, \phi_{1}, \phi_{2}\right\}$, i.e. variance parameters in the transformed model are left as free parameters.
} 
Figure B.1: Nonlinear parameter transformations
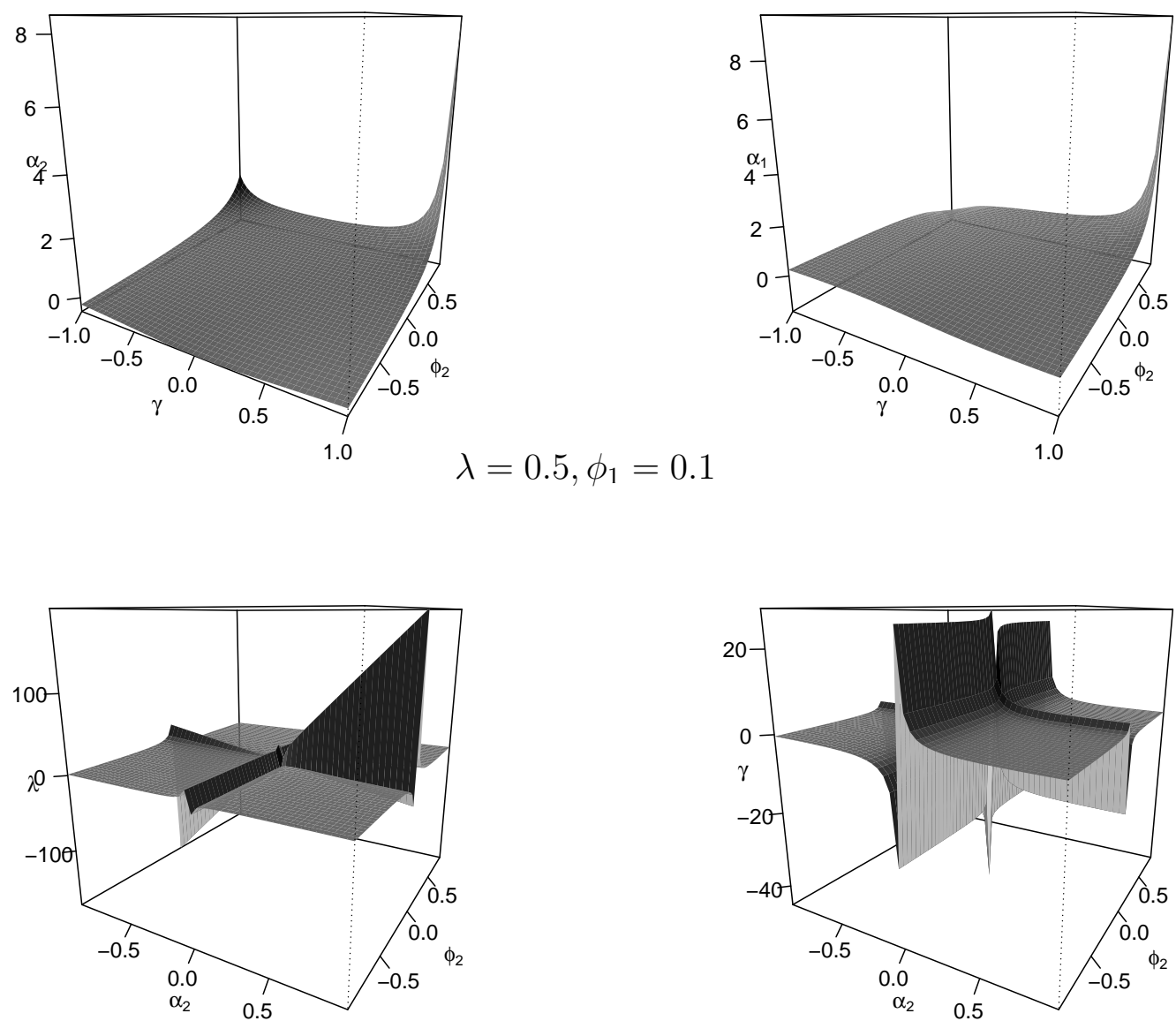

$$
\alpha_{1}=0.5, \phi_{1}=0.1
$$

Note: The top panel presents the implied unrestricted reduced form parameters in (B.2) given structural form parameters in (B.1). The bottom panel presents implied structural form parameters in (B.1) given unrestricted reduced form parameters in (B.2). Parameter transformations are obtained using the RRF restrictions in (B.3). 
and the (observation) variance priors are of inverse-Wishart type ${ }^{8}$

$$
\begin{aligned}
p\left(\lambda, \gamma_{f}, \phi_{1}, \phi_{2} \mid \Sigma\right) & \propto \text { constant for }|\lambda|<1,\left|\gamma_{f}\right|<1,\left|\phi_{1}\right|+\phi_{2}<1,\left|\phi_{2}\right|<1 \\
\Sigma & \sim I W(1,20 \times \tilde{\Sigma})
\end{aligned}
$$

where $I W(\nu, \Psi)$ is the inverse Wishart density with scale $\Psi$ and degrees of freedom $\nu$. It is possible to use economic theory or steady state relationships to construct priors for these parameters, see Del Negro and Schorfheide (2008). We do not follow this approach but let the data information dominate our relatively weak prior information. For the same reason, we perform a prior-predictive analysis and investigate the sensitivity of our posterior results with respect to the prior.

Note that the prior specifications of the observation and state covariances are important in this class of models and for macroeconomic data. Since the sample size is typically small, differentiating the short-run variation in series (the observation variances) from the variation in the long-run (the state variation) can be cumbersome, see Canova (2012). We therefore impose a data based prior on the observation covariances. We first estimate an unrestricted reduced form VAR model using demeaned inflation series and (linear) detrended (log) real marginal cost series, and base the observation variance prior on this covariance estimate, $\tilde{\Sigma}$. This specification imposes smoothness for the estimated levels and trends, and ensures that the state errors do not capture all variation in the observed variables. Second, prior distributions for the extra model parameters stemming from the hybrid models, $\theta_{H}=\left\{\gamma_{b}^{H}, \beta\right\}$ are defined as uniform priors on restricted regions $\left|\gamma_{b}^{H}\right|<1,|\beta|<1$. Third, we define independent inverse-Gamma priors for the state variances

$$
\sigma_{\eta_{1}} \sim I G\left(20,20 \times 10^{-2}\right), \quad \sigma_{\eta_{2}} \sim I G\left(20,20 \times 10^{-3}\right), \quad \sigma_{\eta_{3}} \sim I G\left(1,1 \times 10^{-5}\right),
$$

where $I G(\alpha, \alpha \xi)$ is the inverse-Gamma distribution with shape $\alpha$ and scale $\alpha \xi$. Pa-

\footnotetext{
${ }^{8}$ We experimented with wider truncated uniform densities for the $\lambda$ and $\gamma_{f}$ parameters. The prior truncation does not seem to have a substantial affect on the posterior results.
} 
rameters $\alpha$ and $\xi$ are the a priori number and variance of dummy observations.

Similar to the standard counterparts, the extended NKPC and HNKPC models may also suffer from flat likelihood functions. We therefore set weakly informative priors for the state parameters, such that not all variation in inflation and marginal cost series are captured by the time-varying trends and levels. For example, the number of prior dummy observations for $\sigma_{\eta_{1}}$ and $\sigma_{\eta_{2}}$ is much less than the number of observations to limit the prior information.

The fourth prior distribution we consider is applicable to the NKPC and HNKPC models with level shifts. For these models, we consider a fixed level shift probability of 0.04 . This choice leads to an a priori expected number of shifts of 8 for 200 observations in the sample. Alternatively, this parameter can be estimated together with other model parameters. However, often the limited number of level shifts plague the inference of this parameter. Hence, we set this value, obtained trough an extensive search over intuitive values of this parameter, prior to analysis.

Finally, for the stochastic volatility models, we specify an inverse-gamma prior for the marginal cost variances. For the correlation coefficient, $\rho$, we take an uninformative prior $p(\rho) \propto\left(1-\rho^{2}\right)^{-3 / 2}$, see Çakmaklı et al. (2011).

\section{C.2 Posterior existence and the sampling algorithm}

We summarize the Bayesian inference for the proposed models. An important point regarding the posterior of the structural parameters is the existence of a posterior distribution and its moments, which depends on the number of instruments and the prior. Given one relatively weak instrument (the second lag of the marginal cost series) the posterior will have very fat tails and the existence of the posterior distribution is ensured through priors defined on a bounded region, see Zellner et al. (2013) for a detailed analysis of a linear IV model with small numbers of weak instruments.

The MCMC sampler for the full conditional posterior distribution is based on 
Gibbs sampling with a Metropolis-Hastings step and data augmentation, combining the methodologies in Geman and Geman (1984); Tanner and Wong (1987); Gerlach et al. (2000) and Çakmaklı et al. (2011).

Together with the level specifications of the inflation and real marginal cost series the proposed extended NKPC model takes the following form

$$
\begin{aligned}
\pi_{t}-c_{\pi, t} & =\frac{\lambda}{1-\left(\phi_{1}+\phi_{2} \gamma_{f}\right) \gamma_{f}}\left(z_{t}-c_{z, t}\right)+\frac{\phi_{2} \gamma_{f} \lambda}{1-\left(\phi_{1}+\phi_{2} \gamma_{f}\right) \gamma_{f}}\left(z_{t-1}-c_{z, t-1}\right)+\epsilon_{1, t}, \\
z_{t}-c_{z, t} & =\phi_{1}\left(z_{t-1}-c_{z, t-1}\right)+\phi_{2}\left(z_{t-2}-c_{z, t-2}\right)+\epsilon_{2, t}, \\
c_{\pi, t+1} & =c_{\pi, t}+\kappa_{t} \eta_{1, t+1} \\
c_{z, t+1} & =\mu_{z, t}+c_{z, t}+\eta_{2, t+1}, \\
\mu_{z, t+1} & =\mu_{z, t}+\eta_{3, t+1}
\end{aligned}
$$

where $\left(\epsilon_{1, t}, \epsilon_{2, t}\right)^{\prime} \sim N I D\left(0,\left(\begin{array}{cc}\sigma_{\epsilon_{1}}^{2} & \rho \sigma_{\epsilon_{1}} \sigma_{\epsilon_{2}} \\ \rho \sigma_{\epsilon_{1}} \sigma_{\epsilon_{2}} & \sigma_{\epsilon_{2}}^{2}\end{array}\right)\right),\left(\eta_{1, t}, \eta_{2, t}, \eta_{3, t}\right)^{\prime} \sim N I D\left(0,\left(\begin{array}{ccc}\sigma_{\eta_{1}}^{2} & 0 & 0 \\ 0 & \sigma_{\eta_{2}}^{2} & 0 \\ 0 & 0 & \sigma_{\eta_{3}}^{2}\end{array}\right)\right)$ and the disturbances $\left(\epsilon_{1, t}, \epsilon_{2, t}\right)^{\prime}$ and $\left(\eta_{1, t}, \eta_{2, t}, \eta_{3, t}\right)^{\prime}$ are independent for all $t$.

The NKPC model in (C.3) can be cast into the state-space form as follows

$$
\begin{array}{ll}
Y_{t}=H X_{t}+B U_{t}+\epsilon_{\mathbf{t}}, & \epsilon_{\mathbf{t}} \sim N\left(0, Q_{t}\right) \\
X_{t}=F X_{t-1}+R_{t} \eta_{\mathbf{t}}, & \eta_{\mathbf{t}} \sim N(0, I)
\end{array}
$$

where

$$
\begin{aligned}
& Y_{t}=\left(\begin{array}{c}
\pi_{t} \\
z_{t}
\end{array}\right), \quad X_{t}=\left(\begin{array}{lllll}
c_{\pi, t}, & c_{z, t}, & \mu_{z, t}, & c_{z, t-1}, & c_{z, t-2}
\end{array}\right)^{\prime}, \quad U_{t}=\left(\begin{array}{c}
z_{t} \\
z_{t-1} \\
z_{t-2}
\end{array}\right), \quad \epsilon_{\mathbf{t}}=\left(\begin{array}{c}
\epsilon_{1, t} \\
\epsilon_{2, t}
\end{array}\right), \\
& H=\left(\begin{array}{ccccc}
1 & -\alpha_{1} & 0 & -\alpha_{2} & 0 \\
0 & 1 & 0 & -\phi_{1} & -\phi_{2}
\end{array}\right), B=\left(\begin{array}{ccc}
\alpha_{1} & \alpha_{2} & 0 \\
0 & \phi_{1} & \phi_{2}
\end{array}\right), \quad Q_{t}=\left(\begin{array}{cc}
\sigma_{\epsilon_{1}, t}^{2} & \rho \sigma_{\epsilon_{1}, t} \sigma_{\epsilon_{2}} \\
\rho \sigma_{\epsilon_{1}, t} \sigma_{\epsilon_{2}} & \sigma_{\epsilon_{2}}^{2}
\end{array}\right),
\end{aligned}
$$




$$
F=\left(\begin{array}{ccccc}
1 & 0 & 0 & 0 & 0 \\
0 & 1 & 1 & 0 & 0 \\
0 & 0 & 1 & 0 & 0 \\
0 & 1 & 0 & 0 & 0 \\
0 & 0 & 0 & 1 & 0
\end{array}\right), \quad R_{t}=\left(\begin{array}{ccc}
\kappa_{t} \sigma_{\eta_{1}} & 0 & 0 \\
0 & \sigma_{\eta_{2}} & 0 \\
0 & 0 & \sigma_{\eta_{3}} \\
0 & 0 & 0 \\
0 & 0 & 0
\end{array}\right), \eta_{\mathbf{t}}=\left(\begin{array}{c}
\eta_{1, t} \\
\eta_{2, t} \\
\eta_{3, t}
\end{array}\right),
$$

where $\alpha_{1}=\frac{\lambda}{1-\left(\phi_{1}+\phi_{2} \gamma_{f}\right) \gamma_{f}}$ and $\alpha_{2}=\frac{\lambda \gamma \phi_{2}}{1-\left(\phi_{1}+\phi_{2} \gamma\right) \gamma}$.

Once the state-space form of the model is set as in (C.4) standard inference techniques in state-space models can be carried out. Let $Y_{1: T}=\left(Y_{1}, Y_{2}, \ldots, Y_{T}\right)^{\prime}, X_{1: T}=$ $\left(X_{1}, X_{2}, \ldots, X_{T}\right)^{\prime}, U_{1: T}=\left(U_{1}, U_{2}, \ldots, U_{T}\right)^{\prime}, \sigma_{\epsilon_{1}, 1: T}^{2}=\left(\sigma_{\epsilon_{1}, 1}^{2}, \sigma_{\epsilon_{1}, 2}^{2}, \ldots, \sigma_{\epsilon_{1}, T}^{2}\right)^{\prime}$ and $\theta=$ $\left(\phi_{1}, \phi_{2}, \gamma_{f}, \lambda\right)^{\prime}$. For the most general NKPC model with level shifts and stochastic volatility, the simulation scheme is as follows

1. Initialize the parameters by drawing $\kappa_{t}$ using the prior for level shift probability, $p_{\kappa}$, and by drawing unobserved states $X_{t}, h_{t}$ for $t=1,2, \ldots, T$ from standard normal distribution and conditional on $\kappa_{t}$ for $t=0,1, \ldots, T$. Initialize $m=1$.

2. Sample $\theta^{(m)}$ from $p\left(\theta \mid Y_{1: T}, X_{1: T}, U_{1: T}, R_{1: T}, Q_{1: T}\right)$.

3. Sample $X_{t}^{(m)}$ from $p\left(X_{t} \mid \theta^{(m)}, Y_{1: T}, U_{1: T}, R_{1: T}, Q_{1: T}\right)$ for $t=1,2, \ldots, T$.

4. Sample $h_{t}^{(m)}$ from $p\left(h_{t} \mid X_{1: T}^{(m)}, \theta^{(m)}, Y_{1: T}, U_{1: T}, R_{1: T}, \rho, \sigma_{\epsilon_{2}}^{2}, \sigma_{\eta_{4}}^{2}\right)$ for $t=1,2, \ldots, T$.

5. Sample $\kappa_{t}^{(m)}$ from $p\left(\kappa_{t}^{(m)} \mid \theta^{(m)}, Y_{1: T}, h_{1: T}^{(m)}, U_{1: T}, R_{1: T}, \rho, \sigma_{\epsilon_{2}}^{2}\right)$ for $t=1,2, \ldots, T$.

6. Sample $\sigma_{\eta_{i}}^{2,(m)}$ from $p\left(\sigma_{\eta_{i}}^{2,(m)} \mid X_{1: T}^{(m)}, h_{1: T}^{(m)}, \kappa_{1: T}^{(m)}\right)$ for $i=1,2,3,4$.

7. Sample $\rho^{(m)}$ from from $p\left(\rho^{(m)} \mid X_{1: T}^{(m)}, h_{1: T}^{(m)}, Y_{1: T}, U_{1: T}, \theta^{(m)}, \sigma_{\epsilon_{2}}^{2,(m-1)}\right)$.

8. Sample $\sigma_{\epsilon_{2}}^{2,(m)}$ from from $p\left(\sigma_{\epsilon_{2}}^{2,(m)} \mid \rho^{(m)}, X_{1: T}^{(m)}, h_{1: T}^{(m)}, Y_{1: T}, U_{1: T}, \theta^{(m)}\right)$.

9. Set $m=m+1$, repeat (2)-(9) until $m=M$. 
Steps (3)-(5) are common to many models in the Bayesian state-space framework, see for example Kim and Nelson (1999); Gerlach et al. (2000); Çakmaklı (2012).

\section{Sampling of $\theta$}

Conditional on the states $c_{\pi, t}, c_{z, t}$ and $h_{t}$ for $t=1,2, \ldots, T$, redefining the variables such that $\tilde{\pi}_{t}=\pi_{t}-c_{\pi, t}, \tilde{z}_{t}=z_{t}-c_{z, t}$ and $\varepsilon_{t}=\epsilon_{t} / \exp \left(h_{t} / 2\right)$, the measurement equation in (C.4) can be rewritten as

$$
\begin{aligned}
& \tilde{\pi}_{t}=\frac{\lambda}{1-\left(\phi_{1}+\phi_{2} \gamma_{f}\right) \gamma_{f}} \tilde{z}_{t}+\frac{\phi_{2} \gamma_{f} \lambda}{1-\left(\phi_{1}+\phi_{2} \gamma_{f}\right) \gamma_{f}} \tilde{z}_{t-1}+\varepsilon_{t} \\
& \tilde{z}_{t}=\phi_{1} \tilde{z}_{t-1}+\phi_{2} \tilde{z}_{t-2}+\epsilon_{2, t} .
\end{aligned}
$$

Posterior distributions of the structural parameters under flat priors are non-standard since $z_{t}$ term also is on the right hand side of (C.5) and the model is highly non-linear in parameters. We therefore use two Metropolis Hastings steps to sample these structural parameters, see Metropolis et al. (1953) and Hastings (1970). For sampling $\phi_{1}, \phi_{2}$ conditional on $\lambda, \gamma_{f}$ and other model parameters, the candidate density is a multivariate student- $t$ density on the stationary region with a mode and scale with the posterior mode and scale using only the second equation in (C.5) and 1 degrees of freedom. For sampling $\lambda, \gamma_{f}$ conditional on $\phi_{1}, \phi_{2}$ and other model parameters, the candidate is a uniform density.

\section{Sampling of states, $X_{t}$}

Conditional on the remaining model parameters, drawing $X_{0: T}$ can be implemented using standard Bayesian inference. This constitutes running the Kalman filter first and running a simulation smoother using the filtered values for drawing smoothed states as in Carter and Kohn (1994) and Frühwirth-Schnatter (1994). We start the recursion 
for $t=1, \ldots, T$

$$
\begin{aligned}
X_{t \mid t-1} & =F X_{t-1 \mid t-1} \\
P_{t \mid t-1} & =F P_{t-1 \mid t-1} F^{\prime}+R_{t}^{\prime} R_{t} \\
\eta_{t \mid t-1} & =y_{t}-H X_{t \mid t-1}-B U_{t} \\
\zeta_{t \mid t-1} & =H P_{t \mid t-1} H^{\prime}+Q_{t} \\
K_{t} & =P_{t \mid t-1} H^{\prime} \zeta_{t \mid t-1}^{\prime} \\
X_{t \mid t} & =X_{t \mid t-1}+K_{t} \eta_{t \mid t-1} \\
P_{t \mid t} & =P_{t \mid t-1}-K_{t} H^{\prime} \zeta_{t \mid t-1}^{\prime}
\end{aligned}
$$

and store $X_{t \mid t}$ and $P_{t \mid t}$. The last filtered state $X_{T \mid T}$ and its covariance matrix $P_{T \mid T}$ correspond to the smoothed estimates of the mean and the covariance matrix of the states for period $T$. Having stored all the filtered values, simulation smoother involves the following backward recursions for $t=T-1, \ldots, 1$

$$
\begin{aligned}
\eta_{t+1 \mid t}^{*} & =X_{t+1}-F X_{t \mid t} \\
\zeta_{t+1 \mid t}^{*} & =F P_{t \mid t} F^{\prime}+R_{t+1}^{\prime} R_{t+1} \\
X_{t \mid t, X_{t+1}} & =X_{t \mid t}+P_{t \mid t} F^{\prime} \zeta_{t+1 \mid t}^{*-1} \eta_{t+1 \mid t}^{*} \\
P_{t \mid t, P_{t+1}} & =P_{t \mid t}-P_{t \mid t} F^{\prime} \zeta_{t+1 \mid t}^{*-1} F P_{t \mid t} .
\end{aligned}
$$

Intuitively, the simulation smoother updates the states using the same principle as in the Kalman filter, where at each step filtered values are updated using the smoothed values obtained from backward recursion. For updating the initial states, using the state equation $X_{0 \mid t, X_{1}}=F^{-1} X_{1}$ and $P_{0 \mid t, P_{1}}=F^{-1}\left(P_{1}+R_{1}^{\prime} R_{1}\right) F^{\prime-1}$ can be written for the first observation. Given the mean $X_{t \mid t, X_{t+1}}$ and the covariance matrix $P_{t \mid t, P_{t+1}}$, the states can be sampled from $X_{t} \sim N\left(X_{t \mid t, X_{t+1}}, P_{t \mid t, P_{t+1}}\right)$ for $t=0, \ldots, T$.

\section{Sampling of inflation volatilities, $h_{t}$}

Conditional on the remaining model parameters, we can draw $h_{0: T}$ using standard Bayesian inference as in the case of $X_{t}$. One important difference, however, stems from the logarithmic transformation of the variance in the stochastic volatility model. 
As the transformation concerns the error structure, the square of which follows a $\chi^{2}$ distribution, the system is not Gaussian but follows a log- $\chi^{2}$ distribution. Noticing the properties of $\log -\chi^{2}$ distribution, Kim et al. (1998) and Omori et al. (2007) approximate this distribution using a mixture of Gaussian distributions. Hence, conditional on these mixture components the system remains Gaussian allowing for standard inference outlined above. For details, see Omori et al. (2007). For the estimation of the volatilities in the BVAR-TV-SV model we use the extension of the algorithm following Kastner and Frühwirth-Schnatter (2013) for improving the efficiency of the MCMC algorithm.

\section{Sampling of structural break parameters, $\kappa_{t}$}

Sampling of structural break parameters, $\kappa_{t}$ relies on the conditional posterior of the binary outcomes, i.e. the posterior value in case of a structural break in period $t$ and the posterior value of the case of no structural breaks. However, evaluating this posterior requires one sweep of filtering, which is of order $O(T)$. As this evaluation should be implemented for each period $t$ the resulting procedure would be of order $O\left(T^{2}\right)$. When the number of sample size is large this would result in an infeasible scheme. Gerlach et al. (2000) propose an efficient algorithm for sampling structural break parameters, $\kappa_{t}$, conditional on the observed data, which is still of order $O(T)$. We implement this algorithm for estimation of the structural breaks and refer to Gerlach et al. (2000); Giordani and Kohn (2008) for details.

\section{Sampling of state error variances, $\sigma_{\eta}^{2}$}

Using standard results from a linear regression model with a conjugate prior for the variances in (C.4), it follows that the conditional posterior distribution of $\sigma_{\eta_{i}}^{2}$, with $i=1,2,3,4$ is an inverted Gamma distribution with scale parameter $\Phi_{\eta_{i}}+\sum_{t=1}^{T} \eta_{i, t}^{2}$ and with $T+\nu_{\eta_{i}}$ degrees of freedom for $i=2,3,4$ where $\Phi_{\eta_{i}}$ and $\nu_{\eta_{i}}$ are the scale and degrees of freedom parameters of the prior density. For $i=1$ the parameters of the inverted Gamma distribution becomes $\Phi_{\eta_{1}}+\sum_{t=1}^{T} \kappa_{t} \eta_{1, t}^{2}$ and $\sum_{t=1}^{T} \kappa_{t}+\nu_{\eta_{1}}$.

Sampling of marginal costs variance and correlation coefficient 
To sample the variance of marginal costs and correlation coefficient, we decompose the multivariate normal distribution of $\epsilon_{\mathbf{t}}$ into the conditional distribution of $\epsilon_{2, t}$ given $\epsilon_{1, t}$ and the marginal distribution of $\epsilon_{1, t}$, as in Çakmaklı et al. (2011). This results in

$$
\prod_{t=1}^{T} f\left(\epsilon_{\mathbf{t}}\right)=\prod_{t=1}^{T} \frac{1}{\sigma_{\epsilon_{1, t}}} \phi\left(\frac{\epsilon_{1, t}}{\sigma_{\epsilon_{1, t}}}\right) \frac{1}{\sigma_{\epsilon_{2, t}} \sqrt{\left(1-\rho^{2}\right)}} \phi\left(\frac{\epsilon_{2, t}-\rho \epsilon_{1, t}}{\sigma_{\epsilon_{2, t}}\left(1-\rho^{2}\right)}\right)
$$

Hence, together with prior for the variance in (C.4), variance of the marginal cost series can be sampled using (C.8) by setting up a Metropolis-Hasting step using an

inverted Gamma candidate density with scale parameter $\sum_{t=1}^{T} \epsilon_{2, t}^{2}$ and with $T$ degrees of freedom. To sample $\rho$ from its conditional posterior distribution we can again use (C.8). Conditional on the remaining parameters the posterior becomes

$$
\left(1-\rho^{2}\right)^{-\frac{3}{2}} \prod_{t=1}^{T}\left(\frac{1}{\sqrt{\left(1-\rho^{2}\right)}} \phi\left(\frac{\epsilon_{2, t}-\rho \epsilon_{1, t}}{\sigma_{\epsilon_{2, t}}\left(1-\rho^{2}\right)}\right)\right) .
$$

We can easily implement the griddy Gibbs sampler approach of Ritter and Tanner (1992). Given that $\rho \in(-1,1)$ we can setup a grid in this interval based on the precision we desire about the value of $\rho$.

\section{C.3 Prior-predictive likelihood analysis}

In the proposed models, it is important to assess the effects of the specified prior distributions on the predictive likelihoods. Due to the nonlinear structure of the models, assessing the amount of prior information on the predictive results is not trivial. We present a prior-predictive analysis as in Geweke (2010). For each of the extended NKPC and HNKPC model, we consider 1000 parameter draws from the joint prior distribution and compute the prior predictive likelihoods for the period between 1973-II and 2012I. Hence a comparison of the resulting prior predictions will indicate which model is preferred by the priors. 


\section{Bayesian inference of the extended HNKPC model}

Posterior inference of the HNKPC models with time varying parameters follow similar to section $\mathrm{C}$, using the Gibbs sampler with data augmentation. Together with the level specifications of the inflation and real marginal cost series the proposed extended HNKPC model takes the following form

$$
\begin{aligned}
\pi_{t}-c_{\pi, t} & =\frac{\lambda^{H}}{\left(1-\gamma_{b}^{H} \gamma_{f}^{H}\right)\left(1-\left(\phi_{1}+\phi_{2} \gamma_{f}^{H}\right) \gamma_{f}^{H}\right)}\left(z_{t}-c_{z, t}\right)+\frac{\phi_{2} \gamma_{f}^{H} \lambda^{H}}{\left(1-\gamma_{b}^{H} \gamma_{f}^{H}\right)\left(1-\left(\phi_{1}+\phi_{2} \gamma_{f}^{H}\right) \gamma_{f}^{H}\right)}\left(z_{t-1}-c_{z, t-1}\right) \\
& +\frac{\gamma_{b}^{H} \gamma_{f}^{H}}{\left(1-\gamma_{b}^{H} \gamma_{f}^{H}\right)} \frac{\gamma_{f}^{H}}{1-\gamma_{f}^{H} \beta}\left(\mu_{t}-c_{\pi, t}\right)+\frac{\gamma_{b}^{H}}{\left(1-\gamma_{b}^{H} \gamma_{f}^{H}\right)}\left(\pi_{t-1}-c_{\pi, t-1}\right)+\frac{1}{\left(1-\gamma_{b}^{H} \gamma_{f}^{H}\right)} \epsilon_{1, t}, \\
z_{t}-c_{z, t} & =\phi_{1}\left(z_{t-1}-c_{z, t-1}\right)+\phi_{2}\left(z_{t-2}-c_{z, t-2}\right)+\epsilon_{2, t}, \\
c_{\pi, t+1} & =c_{\pi, t}+\kappa_{t} \eta_{1, t+1}, \\
c_{z, t+1} & =\mu_{z, t}+c_{z, t}+\eta_{2, t+1}, \\
\mu_{z, t+1} & =\mu_{z, t}+\eta_{3, t+1} .
\end{aligned}
$$

This can be cast into the state-space form as in (C.4)

$$
\begin{array}{ll}
Y_{t}=H X_{t}+B U_{t}+\epsilon_{\mathbf{t}}, & \epsilon_{\mathbf{t}} \sim N\left(0, Q_{t}\right) \\
X_{t}=F X_{t-1}+R_{t} \eta_{\mathbf{t}}, & \eta_{\mathbf{t}} \sim N(0, I)
\end{array}
$$

using the following definitions

$$
\begin{aligned}
& Y_{t}=\left(\begin{array}{c}
\pi_{t} \\
z_{t}
\end{array}\right), X_{t}=\left(\begin{array}{cccccc}
c_{\pi, t} & c_{z, t} & \mu_{z, t}, & c_{z, t-1} & c_{z, t-2} & c_{\pi, t-1}
\end{array}\right)^{\prime}, \epsilon_{\mathbf{t}}=\left(\begin{array}{c}
\epsilon_{1, t} \\
\epsilon_{2, t}
\end{array}\right), \\
& U_{t}=\left(\begin{array}{lllll}
z_{t} & z_{t-1} & z_{t-2} & \pi_{t-1} & \mu_{t}
\end{array}\right)^{\prime}, B=\left(\begin{array}{ccccc}
\alpha_{1} & \alpha_{2} & 0 & \alpha_{4} & \alpha_{3} \\
0 & \phi_{1} & \phi_{2} & 0 & 0
\end{array}\right), \\
& H=\left(\begin{array}{cccccc}
1-\alpha_{3} & -\alpha_{1} & 0 & -\alpha_{2} & 0 & -\alpha_{4} \\
0 & 1 & 0 & -\phi_{1} & -\phi_{2} & 0
\end{array}\right), Q_{t}=\left(\begin{array}{cc}
\sigma_{\epsilon_{1}, t}^{2} & \rho \sigma_{\epsilon_{1}, t} \sigma_{\epsilon_{2}} \\
\rho \sigma_{\epsilon_{1}, t} \sigma_{\epsilon_{2}} & \sigma_{\epsilon_{2}}^{2}
\end{array}\right) \text {, }
\end{aligned}
$$




$$
F_{t}=\left(\begin{array}{cccccc}
1 & 0 & 0 & 0 & 0 & 0 \\
0 & 1 & 1 & 0 & 0 & 0 \\
0 & 0 & 1 & 0 & 0 & 0 \\
0 & 1 & 0 & 0 & 0 & 0 \\
0 & 0 & 0 & 1 & 0 & 0 \\
1 & 0 & 0 & 0 & 0 & 0
\end{array}\right), R_{t}=\left(\begin{array}{ccc}
\kappa_{t} \sigma_{\eta_{1}} & 0 & 0 \\
0 & \sigma_{\eta_{2}} & 0 \\
0 & 0 & \sigma_{\eta_{3}} \\
0 & 0 & 0 \\
0 & 0 & 0 \\
0 & 0 & 0
\end{array}\right), \eta_{\mathbf{t}}=\left(\begin{array}{c}
\eta_{1, t} \\
\eta_{2, t} \\
\eta_{3, t}
\end{array}\right),
$$

where parameters $\alpha_{1}, \alpha_{2}, \alpha_{3}, \alpha_{4}$ are defined as functions of the structural form parameters

$$
\begin{aligned}
\alpha_{1} & =\frac{\lambda^{H}}{\left(1-\left(\phi_{1}+\phi_{2} \gamma_{f}^{H}\right) \gamma_{f}^{H}\right)\left(1-\gamma_{b}^{H} \gamma_{f}^{H}\right)}, \quad \alpha_{2}=\frac{\lambda^{H} \gamma_{f}^{H} \phi_{2}}{\left(1-\left(\phi_{1}+\phi_{2} \gamma_{f}^{H}\right) \gamma_{f}^{H}\right)\left(1-\gamma_{b}^{H} \gamma_{f}^{H}\right)}, \\
\alpha_{3} & =\frac{\gamma_{b}^{H} \gamma_{f}^{H}}{\left(1-\gamma_{b}^{H} \gamma_{f}^{H}\right)} \frac{\gamma_{f}^{H}}{\left(1-\gamma_{f}^{H} \beta\right)}, \quad \alpha_{4}=\frac{\gamma_{b}^{H}}{\left(1-\gamma_{b}^{H} \gamma_{f}^{H}\right)} .
\end{aligned}
$$

Given this setup, posterior inference can be carried out using the steps outlined in section C.

\section{E Posterior results for the NKPC models with non- filtered time series}

This section presents additional estimation results for the NKPC models with nonfiltered time series. We summarize the estimated levels, volatilities, breaks and inflation expectations obtained from the NKPC-TV, NKPC-TV-LS and NKPC-TV-LS-SV models. Figure E.1 shows the estimated levels from the three NKPC models. Estimated inflation levels, computed as the posterior mean of the smoothed states, are given in the first row of Figure E.1. Shaded areas around the posterior means represent the 95\% HPDI for the estimated levels. For all three models, estimated inflation levels nicely track the observed inflation. Effects of the level specification are reflected in 
the estimates in various ways. First, when we model inflation level changes as discrete level shifts rather than continuous changes, we observe a relatively smoother pattern in estimated inflation levels. This effect can be seen by comparing the second and first graphs in the first row of Figure E.1. While estimated inflation level in the first graph follows the observed inflation patterns closely, estimated inflation level in the second (and third to a less extent) graph mostly indicates three distinct periods. These periods are the high inflation periods capturing 1970s with a constant inflation level around $1.7 \%$ (quarterly inflation) following a low inflation period in 1960s, and the period after the beginning of $1980 \mathrm{~s}$ with a stable inflation level around $0.5 \%$, see Cecchetti et al. (2007) for similar findings. Second, adding the stochastic volatility together with level shifts results in discrete level shifts in inflation which are more frequent than the model with only level shifts.

The second panel in Figure E.1 presents the estimated levels for the real marginal cost series for all models. A common feature of all these estimates is the smoothness of the estimated levels. In all models, marginal cost series follows a slightly nonlinear trend during the sample period. The estimated slopes of these trends for all models are given in the bottom panel of Figure E.1, together with the 95\% HPDIs. Nonlinearity of the negative trend is reflected in the negative values for the slope of the trend, with an increasing magnitude at the end of the sample. This change in the slope of the trend is accompanied by the increasing uncertainty about the slope. The difference between the models in terms of the estimated marginal cost structures is negligible.

\section{F Posterior results for the HNKPC models with non-filtered time series}

This section presents additional estimation results for the HNKPC models with nonfiltered time series. We summarize the estimated levels, volatilities, breaks and inflation 
Figure E.1: Level, trend and slope estimates from the NKPC models
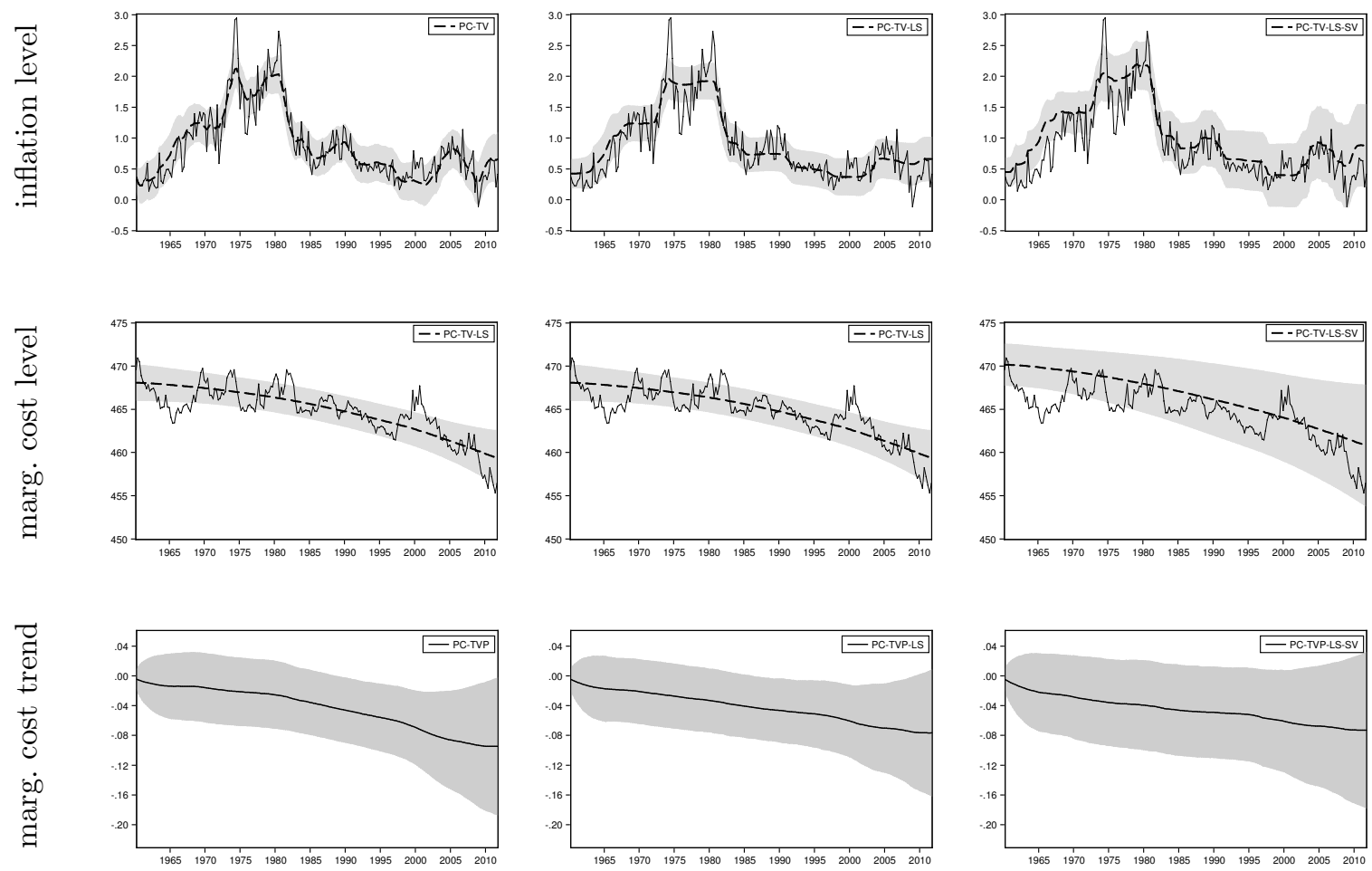

Note: The top panel exhibits estimated inflation levels. The middle and the bottom panels show estimated real marginal cost levels and slopes, respectively. Grey shaded areas correspond to the $95 \%$ HPDI. NKPC-TV refers to the NKPC model with time varying levels and trends. NKPC-TV-LS refers to the NKPC model with time varying levels and trends. NKPC-TV-LS-SV refers to the NKPC model with time varying levels, trends and volatility. HNKPC-TV refers to the Hybrid NKPC model with time varying levels, trends and inflation expectations. HNKPC-TV-LS refers to the HNKPC model with time varying levels, trends and inflation expectations. HNKPC-TV-LS-SV refers to the HNKPC model with time varying levels, trends, inflation expectations and volatility. Results are based on 40000 simulations of which the first 20000 are discarded for burn-in.

expectations obtained from the HNKPC-TV, HNKPC-TV-LS and HNKPC-TV-LS-SV models.

Figure F.1 presents the estimated inflation levels, together with estimated levels and trends of the marginal cost series.

Figure F.2 presents the estimated inflation expectations together with observed survey based inflation expectations. 
Figure F.1: Level, trend and slope estimates from the HNKPC models
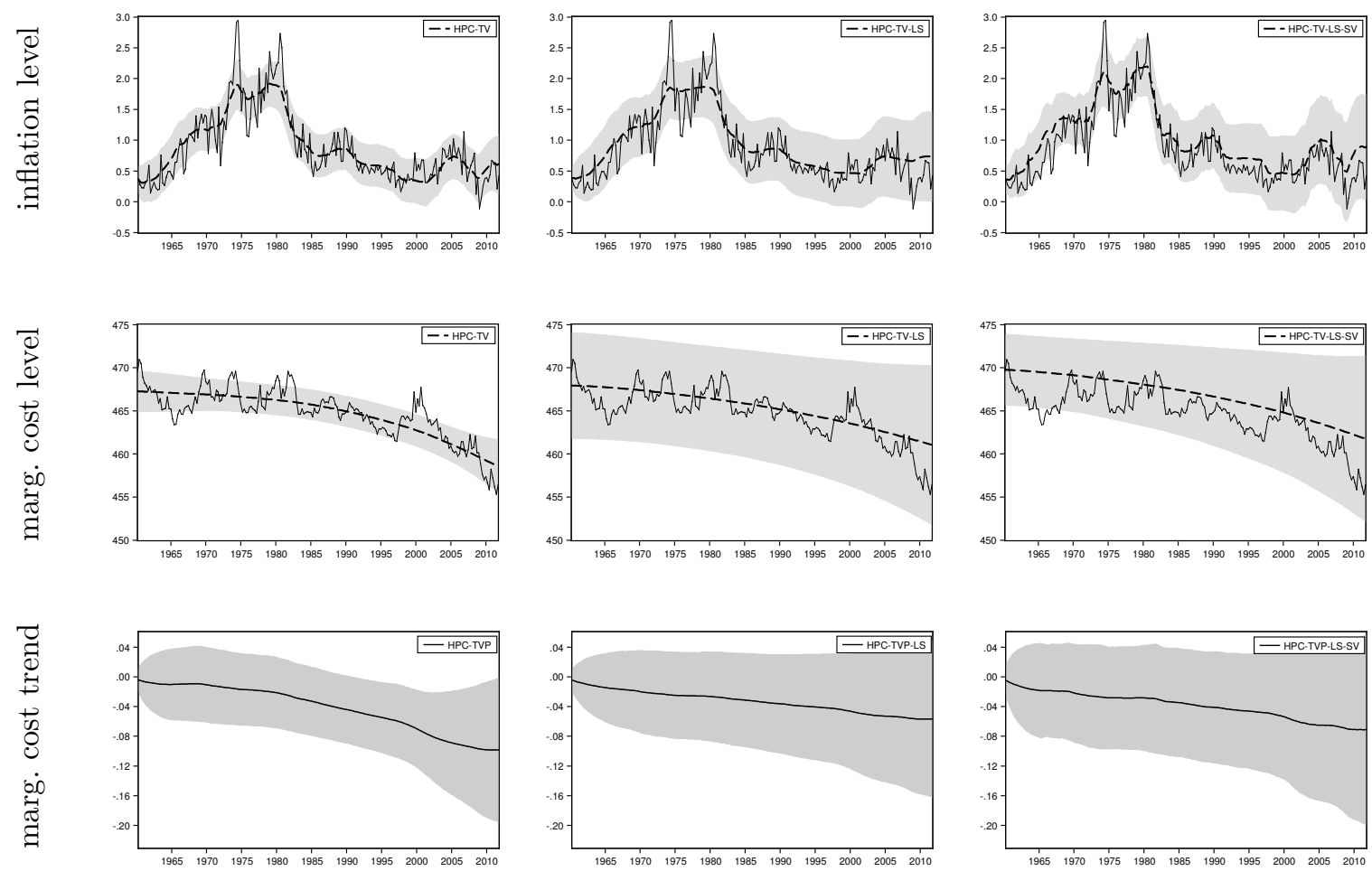

Note: The top panel exhibits estimated inflation levels. The middle and the bottom panels show estimated real marginal cost levels and slopes, respectively. Grey shaded areas correspond to the $95 \%$ HPDI. Results are based on 40000 simulations of which the first 20000 are discarded for burn-in.

Figure F.2: Implied inflation expectations by HNKPC models
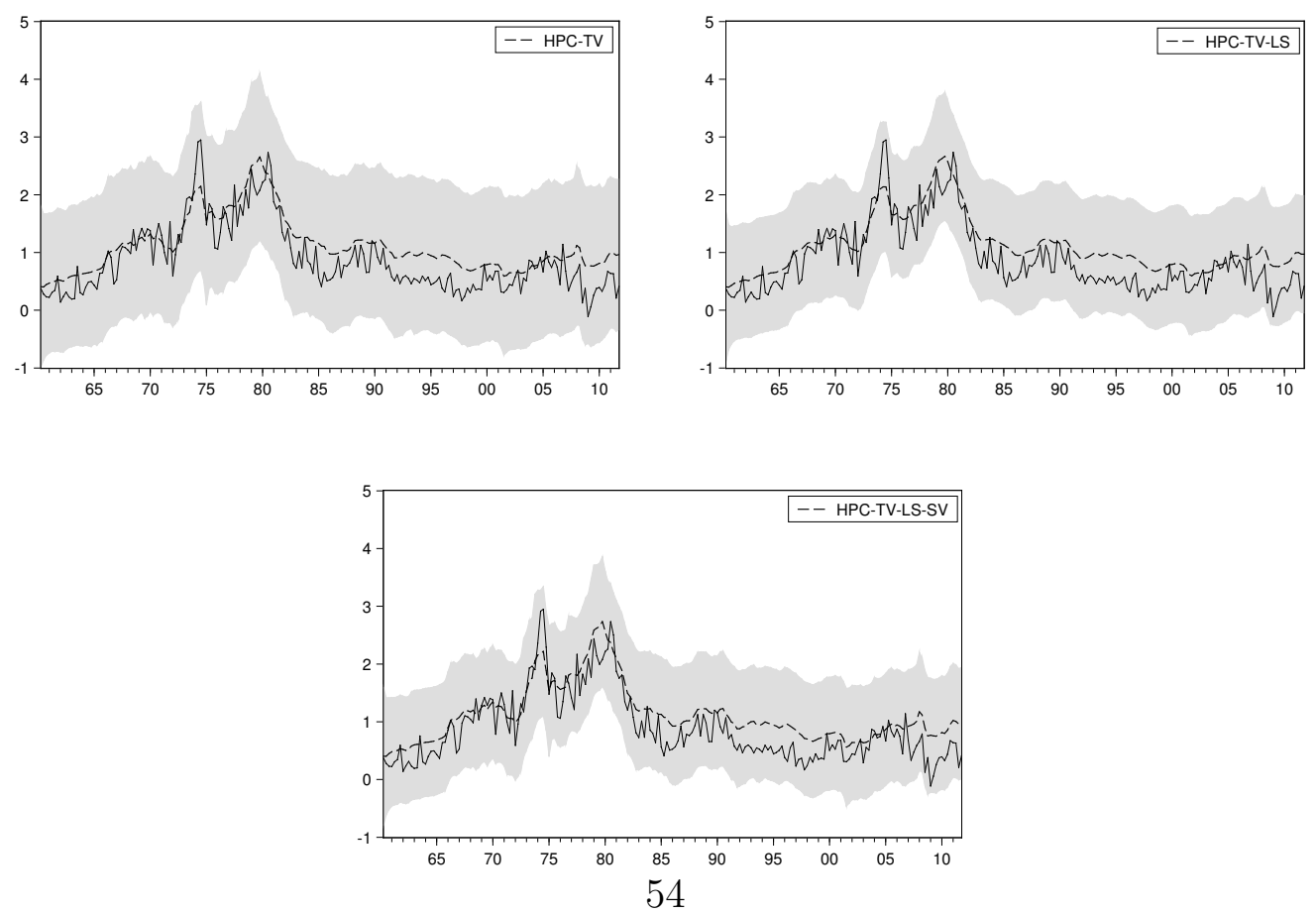

Note: The thick solid lines are the posterior means of inflation expectations from the HNKPC models. The thin solid lines are the observations of inflation expectations from survey data. Grey shaded areas are the $95 \%$ HPDI for estimated inflation expectations. Results are based on 40000 simulations of which the first 20000 are discarded for burn-in. 


\section{G Predicted inflation densities from all proposed models}

This section presents the entire distribution of the inflation predictions for all NKPC and HNKPC models. Predicted inflation densities from all proposed models are presented in Figure G.1. In these figures, the solid lines represent the posterior mean of predicted inflation, and the white areas under the inflation densities show the inflation levels with non-zero posterior probability. For all models we propose, inflation predictions are concentrated around high (low) values during the high (low) inflationary periods. The uncertainty around the inflation predictions are also high for these periods, together with the periods when inflation is subject to a transition to low values around 1980s.

When the observed inflation values are close to the zero bound, the predictive densities indicate disinflationary risk, computed as the fraction of the predictive distribution below zero.

\section{H Prior-predictive likelihoods of proposed models}

Due to the complex model structures in the proposed models, it is important to address the effects of the specified prior distributions on the predictive performances. We therefore perform the prior-predictive analysis outlined in section $\mathrm{C}$ for the extended NKPC models, for the forecast sample analyzed earlier, covering the period between 1973-II and 2012-I. Table H.1 presents the average and cumulative prior predictive likelihoods for the forecast sample. Prior predictive likelihoods, not using the data information and also using weak prior information, naturally perform worse than the predictive results reported in Table I.2. Table H.1 also shows that the adopted prior distributions clearly favor the less parameterized model, NKPC-TV. Moreover, the priors clearly do not favor models with stochastic volatility components. Most importantly, the 'best 
Figure G.1: Predicted inflation densities from NKPC and HNKPC models

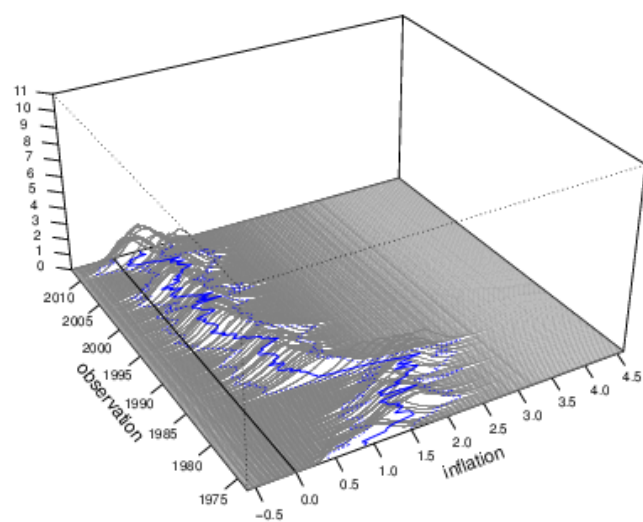

PC-TV

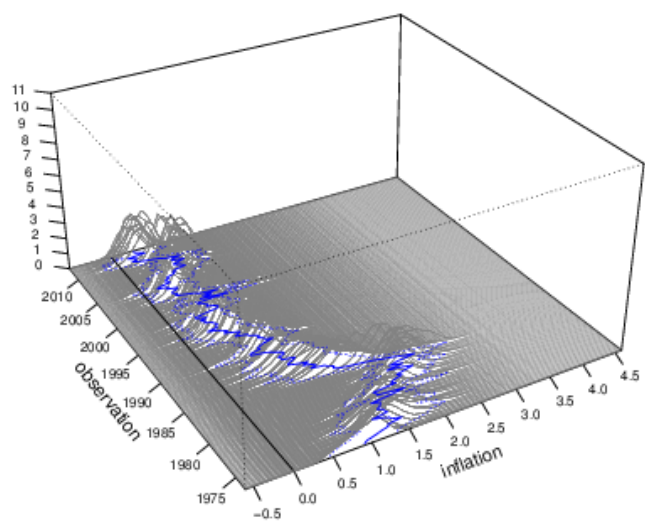

PC-TV-LS

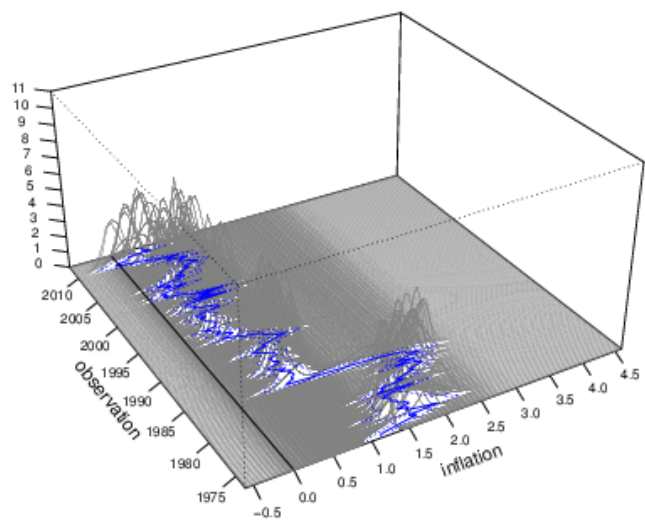

PC-TV-LS-SV

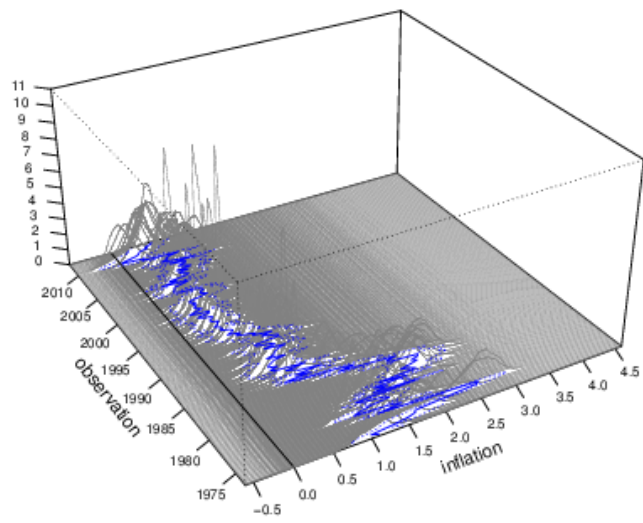

HPC-TV

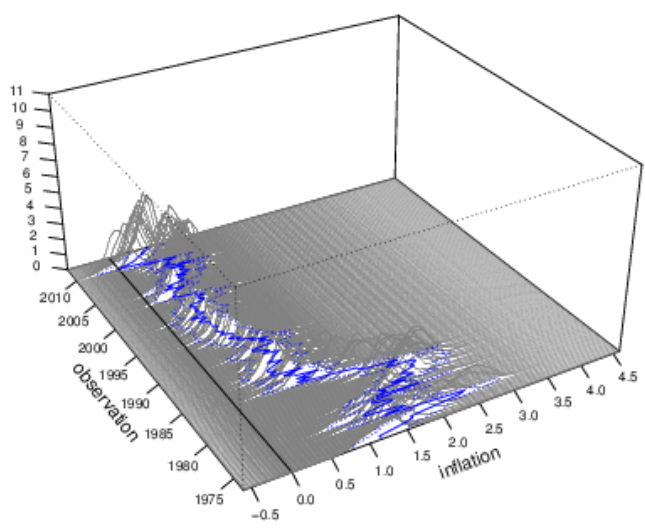

HPC-TV-LS

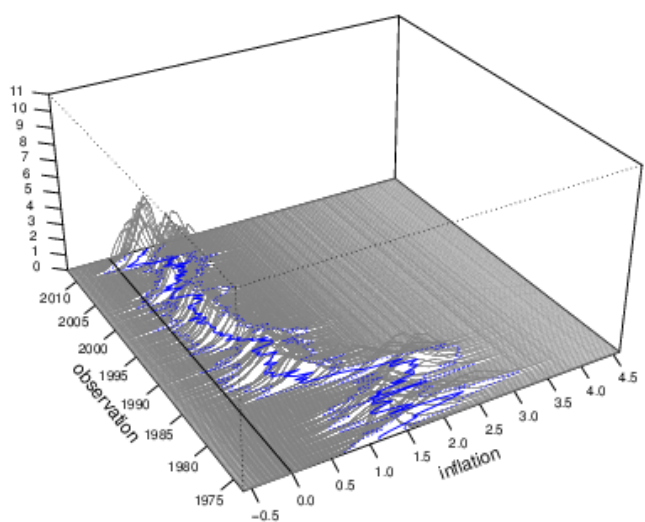

HPC-TV-LS-SV

Note: The figure presents one period ahead predictive distributions of inflation from the NKPC and HNKPC models, for the period between the third quarter of 1973 and the first quarter of 2012. Model abbreviations are as in Figure E.1 . Results are based on 40000 simulations of which the first 20000 are discarded for burn-in. 
performing model' according to the predictive results in Table I.2, HNKPC-TV-LS-SV, is the least favorable one according to the adopted prior distributions using the same forecast sample. We therefore conclude that data information is dominant, and the superior predictive performance of the HNKPC-TV-LS-SV model is not driven by the prior distribution.

Table H.1: Prior-predictive results for the NKPC models

\begin{tabular}{lcc} 
Model & $\begin{array}{c}\text { Average } \\
(\text { Log })\end{array}$ & $\begin{array}{c}\text { Cumulative } \\
\text { Pred. Likelihood }\end{array}$ \\
\hline NKPog) & Pred. Likelihood \\
\hline NKPC-TV-LS & -1.16 & -180.88 \\
NKPC-TV-LS-SV & -1.36 & -210.91 \\
HNKPC-TV & -1.45 & -224.66 \\
HNKPC-TV-LS & -1.28 & -199.22 \\
HNKPC-TV-LS-SV & -1.27 & -197.68 \\
\hline
\end{tabular}

Note: The table reports the prior-predictive performances of all competing models for the prediction sample over the period 1973-II until 2012-I. 'Average (Cumulative) Log Pred. Likelihood' stands for the average (sum) of the natural logarithms of predictive likelihoods. Results are based on 1000 simulations from the joint priors of model parameters. Model abbreviations are as in Table 1 in the paper.

\section{Posterior and predictive results from alternative models for robustness checks}

The proposed NKPC and HNKPC models extend the standard models in several ways. First, both model structures introduce time variation in the long and short run dynamics of inflation and marginal cost series. Second, the introduction and the iterative solution of the expectational mechanisms and the survey data in the extended HNKPC models enables the use of more data information. Furthermore, extended and standard HNKPC models use the additional information from a backward looking component for the inflation series compared to the HNKPC counterparts. According to the predictive results, the most comprehensive model, HNKPC-TV-LS-SV is also the best performing model. However, a deeper analysis is needed in order to see the added predictive gains 
from each of these extensions. In this section we consider several alternative models and their predictive performances to separately address the predictive gains from each of these extensions in the model structure. Table I.1 presents all NKPC and HNKPC model structures we compare to differentiate these effects.

Table I.1: Standard and extended NKPC models

\begin{tabular}{|c|c|c|c|c|}
\hline \multirow{2}{*}{$\begin{array}{l}\text { low/high } \\
\text { frequencies }\end{array}$} & \multicolumn{2}{|c|}{ iterated expectations solution } & \multicolumn{2}{|c|}{ direct expectations data } \\
\hline & NKPC & HNKPC & NKPC & HNKPC \\
\hline linear trend & NKPC-LT & $\mathrm{n} / \mathrm{a}$ * & NKPCS-LT & HNKPCS-LT \\
\hline Hodrick-Prescott filter & NKPC-HP & $\mathrm{n} / \mathrm{a}$ * & NKPCS-HP & HNKPCS-HP \\
\hline time varying levels & NKPC-TV & HNKPC-TV & NKPCS-TV & HNKPCS-TV \\
\hline time varying levels and switching & NKPC-TV-LS & HNKPC-TV-LS & NKPCS-TV-LS & HNKPCS-TV-LS \\
\hline $\begin{array}{l}\text { time varying levels and stochastic } \\
\text { volatility }\end{array}$ & NKPC-TV-SV & HNKPC-TV-SV & NKPCS-TV-SV & HNKPCS-TV-SV \\
\hline $\begin{array}{l}\text { time varying levels, switching and } \\
\text { stochastic volatility }\end{array}$ & $\begin{array}{l}\text { NKPC-TV-LS- } \\
\text { SV }\end{array}$ & $\begin{array}{l}\text { HNKPC-TV-LS- } \\
\text { SV }\end{array}$ & $\begin{array}{l}\text { NKPCS-TV-LS- } \\
\text { SV }\end{array}$ & $\begin{array}{c}\text { HNKPCS-TV-LS- } \\
\text { SV }\end{array}$ \\
\hline
\end{tabular}

Note: The first two columns present the standard and extended (H)NKPC models presented in the main paper, for which expectational mechanisms are solved explicitly. The last two columns present alternative model structures for (H)NKPC models. For these models, we do not iterate inflation expectations in the models, but instead replace them with survey data directly. NKPC(S)-LT (NKPC-HP(S)) refers to the NKPC model where the real marginal cost series is detrended using linear trend (Hodrick-Prescott) filter. For the remaining models real marginal cost series' trend is modeled using local linear trend model. NKPC(S)-TV refers to the NKPC model with time varying inflation levels. NKPC(S)-TV-LS refers to the NKPC model with time varying inflation levels together with level shifts. NKPC(S)-TVSV refers to the NKPC model with time varying inflation levels and stochastic volatility. NKPC(S)-TV-LS-SV refers to the NKPC model with time varying inflation levels together with level shifts and stochastic volatility. HNKPC(S)-TV refers to the Hybrid NKPC model with time varying levels and inflation expectations. HNKPC(S)-TV-LS refers to the HNKPC model with time varying levels together with level shifts and inflation expectations. HNKPC(S)-TV-SV refers to the HNKPC model with time varying levels, inflation expectations and stochastic volatility. HNKPC(S)-TV-LS-SV refers to the HNKPC model with time varying levels together with level shifts, inflation expectations and stochastic volatility.

* Iterative solution of these models without using the survey data does not exist.

The first set of alternative models we consider are the standard NKPC and HNKPC models combined with data from survey expectations, without introducing explicit time variation in the low frequency structure of data but instead demeaning the inflation series, and detrending the marginal cost series prior to analysis. These models are given in the first two rows of the right panel of Table I.1 and are abbreviated by NKPCSLT, NKPCS-HP, HNKPCS-LT and HNKPCS-HP, according to linear detrending or 
HP detrending prior to analysis. The improved predictive performances of NKPCS-LT and NKPCS-HP models compared to the standard NKPC counterparts show predictive gains from incorporating survey expectations in the models. Furthermore, comparing the predictive performances of the HNKPCS-LT and HNKPCS-HP models with the time-varying hybrid models, such as the HNKPC-TV or HNKPC-TV-LS models show the gains from incorporating time variation alone, since all these models use survey data and the backward looking component for inflation.

The second set of alternative models we consider, on the right panel of Table I.1, are NKPC models with time-varying levels, where we incorporate the survey expectations in the model directly rather than solving the model iteratively. These models correspond to (B.1) where the expectation term is replaced by survey expectations. We denote these models by NKPCS-TV, NKPCS-TV-LS and NKPCS-TV-LS-SV, for the time-varying levels, time-varying levels with regimes shifts in inflation and time-varying levels with regime shifts and stochastic volatility component in inflation, respectively. Comparing the predictive results of these models to the HNKPC counterparts provide the predictive gains solely from the HNKPC extension, i.e. they separate the gains from incorporating the backward looking inflation component in the model from the other model extensions.

The third set of alternative models we consider are the HNKPC models using the survey expectations directly, without solving for the expectational mechanisms. We denote these models by HNKPCS-TV, HNKPCS-TV-LS and HNKPCS-TV-LS-SV, for the time-varying levels, time-varying levels with regimes shifts in inflation and timevarying levels with regime shifts and stochastic volatility component, respectively. Comparing the predictive performance of these models with the proposed HNKPC models clarifies the predictive gains from solving for the inflation expectations iteratively in the hybrid models.

The final set of alternative models aim to pinpoint predictive gains from introducing 
level shifts in inflation in the models with a stochastic volatility component. The comparison of the predictive results of models with time-varying levels and stochastic volatility, (H)NKPC-TV-SV, and with level shifts and stochastic volatility, (H)NKPCTV-LS-SV, highlights predictive gains solely from introducing level shifts when changes in inflation volatility are taken into account.

One period ahead MSFE and log marginal likelihoods of these models, together with the standard $(\mathrm{H}) \mathrm{NKPC}$ models and the models proposed in the paper, are given in Table I.2. The prediction results are based on the forecast sample, which covers the period between the second quarter of 1973 and the first quarter of 2012. Comparing the first block and the first two rows of the second block Table I.2, we see that the gains from using survey data inflation are substantial even in the standard NKPC models. In terms of predictive gains, the biggest improvement in predictive likelihoods and the MSFE are achieved with this contribution in the models. However, the predictive performances of these improved models are still far from the more involved models. Hence the gains from the proposed models do not only stem from the inclusion of the survey data information alone.

We also report the predictive gains resulting specifically from introducing timevariation in the inflation and marginal cost series, by comparing the results of the HNKPCS-LT and HNKPCS-HP models with the HNKPC-TV or HNKPC-TV-LS models in the table. The more involved models with time variation clearly perform better according to the predictive results. Especially the difference in marginal likelihoods of these models enables us to conclude that incorporating time variation in the data is also important.

As a third possible reason for predictive gains, we focus on the models with backward looking components. One way to separate the added value from this component is to consider the second block of Table I.2. The prediction results from the NKPC and HNKPC models in this block are very similar, with slight improvements in the 
hybrid models, where the backward looking component is incorporated. Another way to see the effect of the backward looking component is to compare the NKPCS-TV, NKPCS-TV-LS and NKPCS-TV-LS-SV models with HNKPCS-TV, HNKPCS-TV-LS and HNKPCS-TV-LS-SV models, respectively. In all these comparisons, the models without the backward looking component performs slightly better (worse) in terms of MSFE (marginal likelihood), hence the backward looking component does not seem to improve predictive results in general and the improvements in the hybrid models mainly stem from incorporating the survey expectations.

From the considered alternative models, time-varying level models with a stochastic volatility component using survey data directly (NKPCS-TV-LS-SV and HNKPCSTV-LS-SV) clearly perform best. In terms of the predictive likelihoods, these models are also comparable to the 'best performing' model we propose.

A final source of possible predictive gains in the proposed models is the iterative solution of inflation expectations. This comparison is based on the comparison of the models in the third (fourth) block and the fifth (sixth) block of Table I.2, where only the third (fourth) block uses the iterative solution. According to the MSFE, predictive results deteriorate slightly when we solve the system. We find this result rather counterintuitive since the iterative solution is based on the complete model structure. As we show briefly, despite this slight increase in the predictive performances, models without the iterative solutions suffer from identification issues.

We next focus on changes in parameter estimates for the alternative models proposed in this section. Table I.3 presents the parameter estimates for all alternative models. Despite the predictive gains from these alternative models, parameter estimates are rather different from those obtained from the proposed models. Specifically for the hybrid models considered, uncertainty in posterior distributions increase substantially if the iterative model solution is not used. Furthermore, posterior densities of some parameters are quite irregular in most of these models which use expectations 
data directly. Figure I.1 shows this irregularity for the HNKPCS-TV model, parameters $\lambda^{(H)}, \gamma_{b}^{(H)}$ and $\gamma_{f}^{(H)}$. The bimodality problem in posterior densities is most apperant in the NKPC slope, $\lambda_{b}^{(H)}$. Furthermore, the backward looking component $\gamma_{b}^{(H)}$ is spread over a wide region with multiple modes. Similar results hold for the remaining alternative models which make use of the survey expectations data directly. We therefore conclude that replacing the expectational term in the $(\mathrm{H}) \mathrm{NKPC}$ models with survey expectations deteriorate posterior inference compared to the iterative solution of these expectational terms.

Figure I.1: Posterior density of $\lambda^{(H)}, \gamma_{b}^{(H)}$ and $\gamma_{f}^{(H)}$ from the HNKPCS-TV model
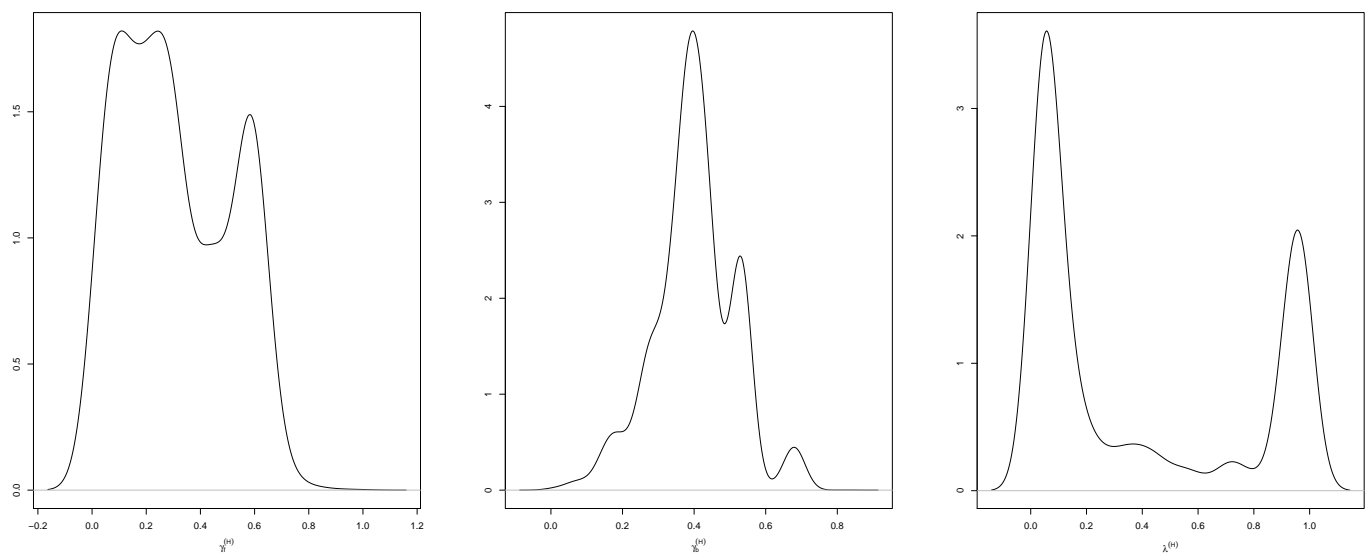

Note: The figure presents posterior densities of parameters from the HNKPCS-TV model. Model abbreviations are based on Table I.1. Results are based on 40000 simulations of which the first 20000 are discarded for burn-in. 
Table I.2: Predictive performance of additional NKPC models

\begin{tabular}{lrc} 
Model & $\begin{array}{c}\text { (Log) Marg. } \\
\text { Likelihood }\end{array}$ & $\begin{array}{c}\text { MSFE } \\
\text { 1 period ahead }\end{array}$ \\
\hline NKPC-LT & -139.327 & 0.353 \\
NKPC-HP & -157.195 & 0.458 \\
\hline NKPCS-LT & -79.141 & 0.105 \\
NKPCS-HP & -85.397 & 0.130 \\
HNKPCS-LT & -81.047 & 0.105 \\
HNKPCS-HP & -85.200 & 0.119 \\
\hline NKPC-TV & -46.162 & 0.142 \\
NKPC-TV-LS & -61.972 & 0.138 \\
NKPC-TV-SV & -22.761 & 0.134 \\
NKPC-TV-LS-SV & -33.476 & 0.126 \\
\hline HNKPC-TV & -36.385 & 0.123 \\
HNKPC-TV-LS & -35.052 & 0.105 \\
HNKPC-TV-SV & -19.695 & 0.106 \\
HNKPC-TV-LS-SV & -18.150 & 0.091 \\
\hline NKPCS-TV & -34.407 & 0.129 \\
NKPCS-TV-LS & -32.004 & 0.099 \\
NKPCS-TV-LS-SV & -15.390 & 0.092 \\
\hline HNKPCS-TV & -40.465 & 0.176 \\
HNKPCS-TV-LS & -38.082 & 0.297 \\
HNKPCS-TV-LS-SV & -12.977 & 0.139 \\
\hline BVAR (constant) & -166.226 & 0.085 \\
BVAR-TV-SV & -97.980 & 0.100 \\
SW2007 & -78.033 & 0.168
\end{tabular}

Note: The table reports the predictive performances of alternative models for the period between the second quarter of 1973 and the first quarter of 2012. '(Log) Marg. Likelihood' stands for the natural logarithm of the marginal likelihoods. 'MSFE' stands for the Mean Squared Forecast Error. Marginal likelihood values in the first column are calculated as the sum of the predictive likelihood values in the prediction sample. Results are based on 10000 simulations of which the first 5000 are discarded for burn-in. Model abbreviations are based on Table I.1. BVAR (constant) denotes the BVAR model with 2 lags and with constant parameters. 'BVAR-TV-SV' denotes the 'BVAR' model with 2 lags, time varying levels for both series and stochastic volatility for inflation. 'SW2007 stands for the model proposed by Stock and Watson (2007). 


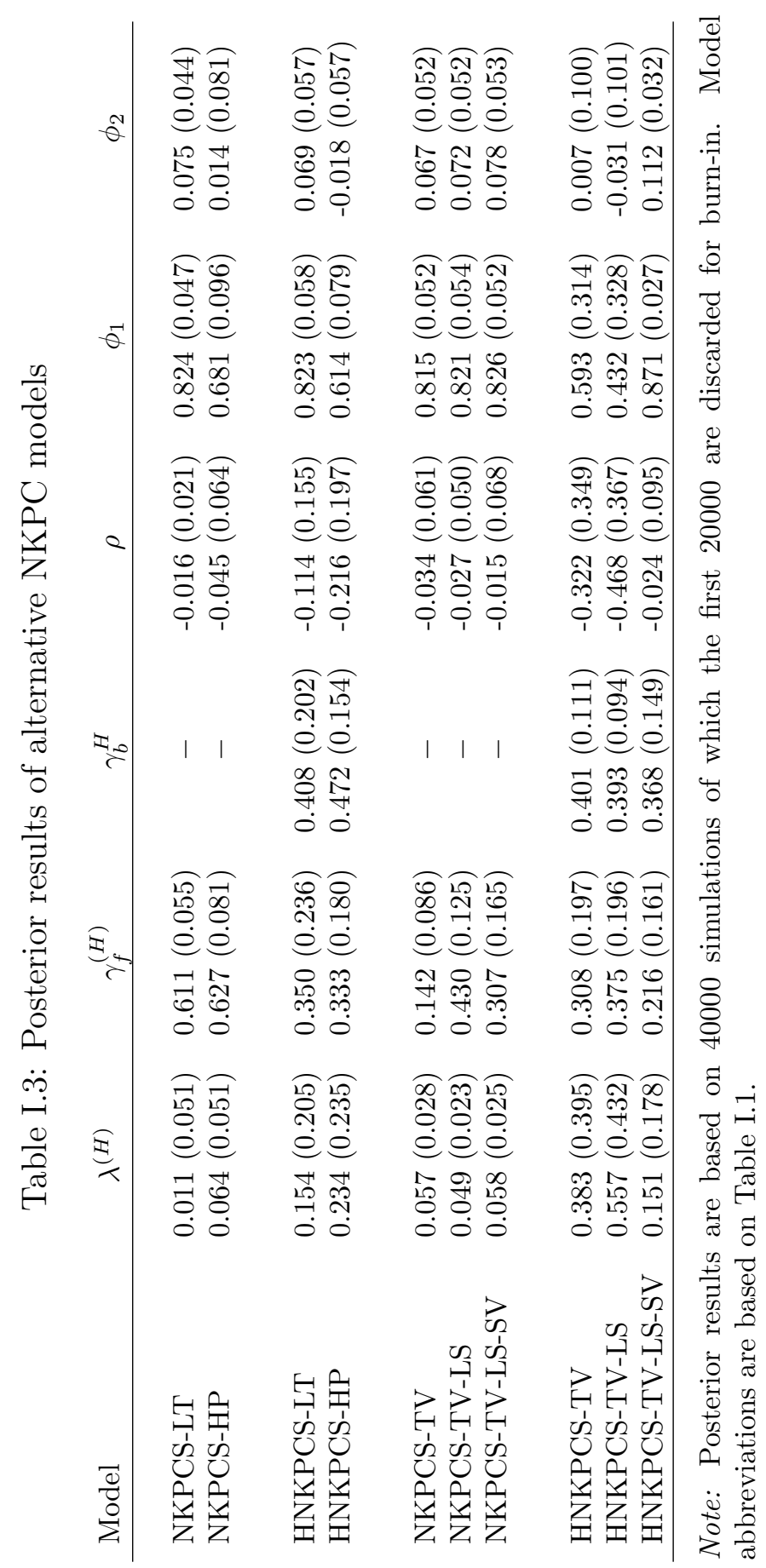


To conclude, predictive gains obtained from including the survey expectations in the models are substantial and incorporating the low and high frequency data movements in the model is crucial. These two conclusions are in line with Faust and Wright (2013), who consider a large set of alternative models for inflation forecasting, including unrestricted reduced form models, and compare their forecast performances based on MSFE. Our model incorporates both these features in the NKPC model structure. Third, once survey data and time variation are included in the model, there are still additional predictive gains from the backward looking component in the hybrid models.

\section{J Modeling inflation expectations using unobserved components}

The HNKPC models implicitly assume that survey based inflation expectations capture 'real' inflation expectations for the next period accurately. However, survey expectations are likely to reflect real inflation expectations with a measurement error. In this section we extend the HNKPC model by including a latent variable for unobserved inflation expectations, aiming to account for the possibility of measurement errors in survey expectations. Specifically, we propose an adaptive rule under which inflation expectations partially adjust to survey expectations at each period:

$$
S_{t+1}=\mu_{t+1}+\beta_{S}\left(S_{t}-\mu_{t}\right)+\eta_{S, t+1}
$$

where $\left|\beta_{S}\right|<1$ and $\mu_{t}$ is the survey observation for inflation expectation at time $t$. This adaptive rule implies that unobserved inflation expectations converge to the survey based expectations in the long run. Given the restriction on parameter $\beta_{S}$, one can

solve (J.1) for $S_{t}$ and obtain $S_{t}=\mu_{t}+\sum_{j=0}^{\infty} \beta_{S}^{j} \eta_{S, t-j}$. This specification allows for the interpretation that expected inflation is equal to the survey values with a measurement error that is specified as an infinite moving average with declining weights. 
We next consider the HNKPC model given the specified adaptive rule for the unobserved inflation expectations. Notice that we can factorize the expectation term in equation (9) in the main text of the paper, $E_{t}\left(\tilde{\pi}_{t+k}\right)$, into two parts related to the measurement error and the relation between survey based expectations and long run expectations, as $E_{t}\left(\tilde{\pi}_{t+k}\right)=E_{t}\left(S_{t+k-1}-\mu_{t+k-1}\right)+E_{t}\left(\mu_{t+k-1}-c_{\pi, t+k}\right)$. Then the weighted sum of expectations in equation (9) in the paper becomes

$$
\sum_{k=1}^{\infty} \gamma_{f}^{k} E_{t}\left(\tilde{\pi}_{t+k}\right)=\sum_{k=1}^{\infty} \gamma_{f}^{k} E_{t}\left(S_{t+k-1}-\mu_{t+k-1}\right)+\sum_{k=1}^{\infty} \gamma_{f}^{k} E_{t}\left(\mu_{t+k-1}-c_{\pi, t+k}\right)
$$

The first part of the summation, $\sum_{k=1}^{\infty} \gamma_{f}^{k} E_{t}\left(S_{t+k-1}-\mu_{t+k-1}\right)$, is related to the measurement error and can be computed from (J.1). For the second part of the summation, $\sum_{k=1}^{\infty} \gamma_{f}^{k} E_{t}\left(\mu_{t+k-1}-c_{\pi, t+k}\right)$, we specify a similar partial adjustment process as the process specified in the paper $\mu_{t}-c_{\pi, t+1}=\beta_{\mu}\left(\mu_{t-1}-c_{\pi, t}\right)+\eta_{\mu, t+1}$. The partial adjustment mechanism implies that the further one gets into the future the smaller will be the difference between short and long run inflation expectations. Estimates of $\beta_{\mu}$ will indicate the empirical speed of adjustment. For instance, for a value of the posterior mean of $\beta_{\mu}$ equal to 0.5 it follows that within a few periods one has almost complete adjustment.

Replacing the infinite sum of expectations of inflation deviations using the two specifications for the measurement error and for the deviation of the survey expectations from the long run inflation expectations in (J.2), the HNKPC model becomes

$$
\begin{aligned}
\pi_{t}-c_{\pi, t} & =\frac{\lambda^{H}}{\left(1-\gamma_{b}^{H} \gamma_{f}^{H}\right)\left(1-\left(\phi_{1}+\phi_{2} \gamma_{f}^{H}\right) \gamma_{f}^{H}\right)}\left(z_{t}-c_{z, t}\right)+\frac{\phi_{2} \gamma_{f}^{H} \lambda^{H}}{\left(1-\gamma_{b}^{H} \gamma_{f}^{H}\right)\left(1-\left(\phi_{1}+\phi_{2} \gamma_{f}^{H}\right) \gamma_{f}^{H}\right)}\left(z_{t-1}-c_{z, t-1}\right) \\
& +\frac{\gamma_{b}^{H} \gamma_{f}^{H}}{\left(1-\gamma_{b}^{H} \gamma_{f}^{H}\right)}\left(\frac{\gamma_{f}^{H}}{1-\gamma_{f}^{H} \beta_{S}}\left(S_{t}-\mu_{t}\right)+\frac{\gamma_{f}^{H}}{1-\gamma_{f}^{H} \beta_{\mu}}\left(\mu_{t}-c_{\pi, t}\right)\right) \\
& +\frac{\gamma_{b}^{H}}{\left(1-\gamma_{b}^{H} \gamma_{f}^{H}\right)}\left(\pi_{t-1}-c_{\pi, t-1}\right)+\frac{1}{\left(1-\gamma_{b}^{H} \gamma_{f}^{H}\right)} \epsilon_{1, t}, \\
z_{t}-c_{z, t} & =\phi_{1}\left(z_{t-1}-c_{z, t-1}\right)+\phi_{2}\left(z_{t-2}-c_{z, t-2}\right)+\epsilon_{2, t} .
\end{aligned}
$$

Notice that if the speed of adjustment for both specifications are equal, i.e. $\beta_{S}=\beta_{\mu}$, 
then the HNKPC reduces to

$$
\begin{aligned}
\pi_{t}-c_{\pi, t} & =\frac{\lambda^{H}}{\left(1-\gamma_{b}^{H} \gamma_{f}^{H}\right)\left(1-\left(\phi_{1}+\phi_{2} \gamma_{f}^{H}\right) \gamma_{f}^{H}\right)}\left(z_{t}-c_{z, t}\right)+\frac{\phi_{2} \gamma_{f}^{H} \lambda^{H}}{\left(1-\gamma_{b}^{H} \gamma_{f}^{H}\right)\left(1-\left(\phi_{1}+\phi_{2} \gamma_{f}^{H}\right) \gamma_{f}^{H}\right)}\left(z_{t-1}-c_{z, t-1}\right) \\
& +\frac{\gamma_{b}^{H} \gamma_{f}^{H}}{\left(1-\gamma_{b}^{H} \gamma_{f}^{H}\right)} \frac{\gamma_{f}^{H}}{1-\gamma_{f}^{H} \beta_{S}}\left(S_{t}-c_{\pi, t}\right)+\frac{\gamma_{b}^{H}}{\left(1-\gamma_{b}^{H} \gamma_{f}^{H}\right)}\left(\pi_{t-1}-c_{\pi, t-1}\right)+\frac{1}{\left(1-\gamma_{b}^{H} \gamma_{f}^{H}\right)} \epsilon_{1, t}, \\
z_{t}-c_{z, t} & =\phi_{1}\left(z_{t-1}-c_{z, t-1}\right)+\phi_{2}\left(z_{t-2}-c_{z, t-2}\right)+\epsilon_{2, t} .
\end{aligned}
$$

We next compare the models specified in (J.3) and in (J.4) with a HNKPC-TV parametrization in terms of their forecast performances. For the forecast sample considered in the paper, the cumulative predictive likelihood for the HNKPC-TV model in (J.3) is -36.19 while for the model in (J.4) this value is -36.44 . The cumulative predictive likelihood values for the HNKPC-TV model with and without the restriction $\beta_{S}=\beta_{\mu}$ indicate that this restriction is statistically valid as the difference between the likelihood values are very small. Following this evidence we display the parameter estimates of all extended HNKPC models using the expectation specification in (J.4) in Table J.1. We further report the cumulative predictive likelihood values and 1 step ahead MSFE for these models in Table J.2.

Table J.1: Posterior results of HNKPC models with unobserved inflation expectations

\begin{tabular}{lccccccc} 
Model & $\lambda^{H}$ & $\gamma_{f}^{H}$ & $\gamma_{b}^{H}$ & $\beta_{S}$ & $\rho$ & $\phi_{1}$ & $\phi_{2}$ \\
\hline HNKPC-TV & $0.05(0.03)$ & $0.02(0.03)$ & $0.38(0.14)$ & $0.49(0.28)$ & $0.01(0.06)$ & $0.81(0.05)$ & $0.07(0.05)$ \\
HNKPC-TV-LS & $0.04(0.02)$ & $0.01(0.01)$ & $0.49(0.11)$ & $0.52(0.18)$ & $0.02(0.01)$ & $0.79(0.09)$ & $0.19(0.08)$ \\
HNKPC-TV-LS-SV & $0.06(0.02)$ & $0.04(0.10)$ & $0.22(0.12)$ & $0.44(0.24)$ & $-0.01(0.01)$ & $0.82(0.05)$ & $0.15(0.04)$ \\
\hline
\end{tabular}

Note: The table presents posterior means and standard deviations (in parentheses) of parameters for the competing HNKPC type models estimated for quarterly inflation and real marginal costs over the period 1960-I until 2012-I. $\lambda^{H}$ and $\gamma_{f}^{H}$ are the slope of the Phillips curve and the coefficient of inflation expectations in HNKPC model in (J.4). $\gamma_{b}^{H}$ is the coefficient of the backward looking component in the HNKPC model in (J.4). $\beta_{S}$ is the autoregressive parameter for the deviation of inflation expectations, as used in (J.4). $\rho$ is the correlation coefficient of the residuals $\epsilon_{1}$ and $\epsilon_{2}$. $\phi_{1}$ and $\phi_{2}$ are the autoregressive parameters for the real marginal cost specification. Posterior results are based on 40000 simulations of which the first 20000 are discarded for burn-in. Model abbreviations are as in Table 1 in the paper.

Results are very similar to the corresponding table in the paper (Table 2), thus, we 
Table J.2: Predictive performance of HNKPC models with unobserved inflation expectations

\begin{tabular}{lcc} 
Model & $\begin{array}{c}\text { Cumulative } \\
(\text { Log })\end{array}$ & $\begin{array}{c}\text { MSFE } \\
\text { Pred. Likelihood }\end{array}$ \\
\hline HNKPC-TV & -36.44 & 0.12 \\
HNKPC-TV-LS & -35.77 & 0.09 \\
HNKPC-TV-LS-SV & -17.96 & 0.09 \\
\hline
\end{tabular}

Note: The table reports the predictive performances of competing models for the prediction sample over the period 1973-II until 2012-I. 'Cumulative (Log) Pred. Likelihood' stands for the sum of the natural logarithms of predictive likelihoods. 'MSFE' stands for the Mean Squared Forecast Error. Results are based on 10000 simulations of which the first 5000 are discarded for burn-in. Remaining abbreviations are as in Table 1 in the paper.

conclude that the effect of the measurement errors in survey expectations is negligible.

\section{$\mathrm{K}$ Analysis of cointegration in inflation and marginal cost levels}

The models in the paper considered rely on the implicit assumption of the absence of a long-run cointegrating relationship between the inflation and marginal cost series. We assess whether this assumption is plausible for the U.S. data. For this reason, we consider the NKPC-TV model that provides the unobserved levels of both series at each posterior draw. For each of these obtained posterior draws, we perform a simple two-step analysis to check the existence of the cointegrating relationship, which can be seen as a Bayesian extension of the method of Engle and Granger (1987).

We perform a two step analysis, where in the first step we obtain the residuals from the regression of the estimated level of inflation on a constant and the estimated level of marginal costs, for each posterior draw. This implies that we take the estimation uncertainty in the analysis into account. Next, we obtain the posterior distribution of the autoregressive parameter, $\rho$, for each set of residuals from the following regression 
using flat priors on the identified region $\rho \in[-1,1]$

$$
\Delta \hat{\epsilon}_{t}=\rho \hat{\epsilon}_{t-1}+\eta_{t}, \quad \eta_{t} \sim N I D\left(0, \sigma^{2}\right)
$$

where $\hat{\epsilon}_{t}$ denotes the residuals from the first stage, and $\rho=0$ implies that there is no cointegrating relationship between the series. An HPDI including the value of 0 indicates that a cointegrating relation between inflation and marginal cost is unlikely.

We compute the mean and the quantiles of these individual densities using 5000 posterior draws, and report the average values of the mean and the quantiles of $\rho$ based on 3000 simulations. These results are presented in Figure K.1. Posterior means of parameter $\rho$ are around 0 for all posterior draws of inflation and marginal cost levels, and the $80 \%$ an $90 \%$ percent quantiles of the distribution are around 0 as well. Hence this simulation experiment does not indicate a cointegrating relationship between the inflation and marginal cost levels. This pattern is also found for other TV-NKPC models we considered for the U.S. data, but these results are not reported for the sake of brevity. We conclude that the underlying assumption of "no cointegrating relationship' is found to be feasible for the NKPC models we consider.

Figure K.1: Cointegration analysis for the marginal costs and inflation series

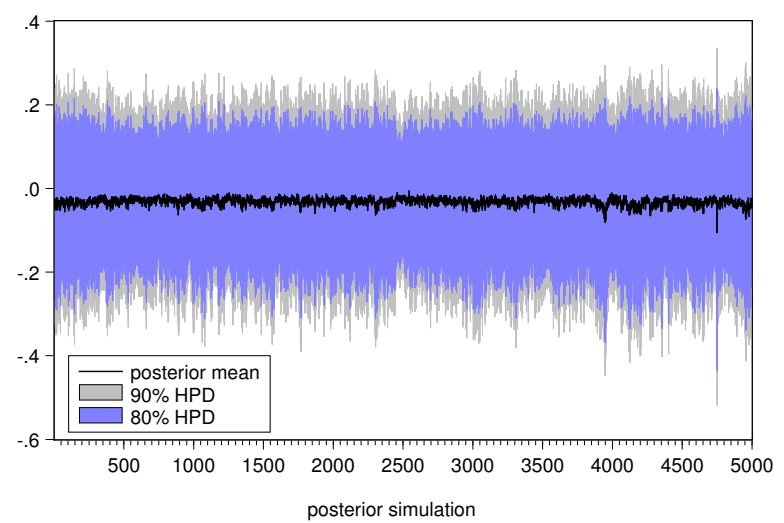

Note: The figure presents the posterior means and quantiles of the $\rho$ parameter from $5 \times 10^{3}$ posterior draws from the NKPC-TV models, where for each draw, the the reported values are calculated using 3000 simulations. $\rho=0$ implies that there is no cointegrating relationship between the series. 